Groups Geom. Dyn. 7 (2013), 977-1011

DOI $10.4171 / \mathrm{GGD} / 213$
Groups, Geometry, and Dynamics

(C) European Mathematical Society

\title{
Filling inequalities for nilpotent groups through approximations
}

\author{
Robert Young
}

\begin{abstract}
We bound the higher-order Dehn functions and other filling invariants of certain Carnot groups using approximation techniques. These groups include the higher-dimensional Heisenberg groups, jet groups, and central products of 2-step nilpotent groups. Some consequences of this work are a construction of groups with arbitrarily large nilpotency class that have Euclidean $n$-dimensional filling volume functions, and a proof of part of a conjecture of Gromov on the higher-order filling functions of the higher-dimensional Heisenberg groups.
\end{abstract}

Mathematics Subject Classification (2010). 20F65, 20 F18.

Keywords. Dehn function, Heisenberg group, filling inequalities, nilpotent groups.

\section{Introduction}

A filling invariant is an invariant of a space which measures the difficulty of finding a disc or chain with a specified boundary. These invariants are studied in geometric group theory because their asymptotic growth rates describe the large-scale geometry of a group or a space.

The primary example of a filling invariant is the Dehn function of a space, which describes the area necessary to fill a closed curve with a disc. This is an important invariant of geometric group theory because if $G$ is a group that acts geometrically (cocompactly, properly discontinously, and by isometries) on a space, then filling a closed curve in the space with a disc corresponds to reducing a word in the group to the identity using relators. Thus, the Dehn function gives a way to connect the combinatorial group theory of a group to the geometry of spaces that it acts on.

The Dehn function can be generalized to the $k$-th order Dehn function $\delta_{X}^{k}(V)$ of a space $X$, which bounds the volume necessary to fill a $k$-sphere in $X$ of volume $\leq V$ by a $k+1$-disc. Since spheres and cycles can have much more complicated metrics than closed curves, much less is known about these higher-order filling invariants than about Dehn functions. In this paper, we will give methods for bounding the higher-order Dehn functions of certain nilpotent groups based on techniques from geometric measure theory. 
These techniques generalize work of Gromov on the Dehn functions of nilpotent groups. Gromov proved that Lipschitz maps of spheres into nilpotent groups which have sufficiently many horizontal maps (i.e., groups which satisfy an appropriate flexibility condition) can be extended to Lipschitz maps of balls [14]. In particular, if a group satisfies the conditions, a closed curve in the group of length $\ell$ can be filled by a Lipschitz map of a disc with Lipschitz constant $O(\ell)$, providing a Dehn function estimate [13]; this works because a closed curve can be replaced by a Lipschitz map of a circle with Lipschitz constant $\ell$. These techniques do not, however, give good higher-order Dehn function estimates, since a map of a sphere with bounded volume may have an arbitrarily large Lipschitz constant.

We will solve this problem by using approximation techniques and combining information from multiple scales. We use the Federer-Fleming Deformation Theorem to approximate singular cycles in a group by simplicial cycles; by rescaling the cycles, we produce approximations which use larger or smaller simplices. By linking these approximations together, we get a filling of the original cycle. Under appropriate conditions, the approximations and the filling all have small volumes.

This method constructs a filling of a sphere out of scalings of a finite set of pieces; indeed, from scalings of the simplices of a triangulation satisfying certain properties. Any way of constructing such a triangulation gives a bound on the Dehn functions or higher-order Dehn functions. We will construct these triangulations in two different ways: we will use holonomic maps in jet spaces to construct fillings in jet groups, and we will show that certain presentations of nilpotent groups can be used to prove quadratic Dehn functions.

We prove bounds for two families of groups. The first family consists of the jet groups. These groups have been used as examples of non-rigid Carnot groups by Warhurst [20] and as a family of quadratically presented Lie algebras by Chen [7]. We show that these groups have many horizontal submanifolds and use these submanifolds to construct triangulations; this leads to Euclidean bounds on their filling functions. Specifically, we show:

Theorem 1. For any $m>0, k>0$, the group $J^{m}\left(\mathbb{R}^{k}\right)$, when endowed with a left-invariant Riemannian metric, has

$$
\mathrm{FV}^{i}(V) \sim \delta^{i-1} \sim V^{\frac{i}{i-1}}
$$

for $i \leq k$, and

$$
\mathrm{FV}^{k+1}(V) \sim \delta^{k} \sim V^{\frac{k+m+1}{k}} .
$$

Here $J^{m}\left(\mathbb{R}^{k}\right)$ is a $(k+1)$-step nilpotent group based on the $m$-jet bundle of $\mathbb{R}^{k}$. For $i \leq k$, these are the same filling inequalities as for Euclidean space, so we call these bounds Euclidean. This gives examples of groups of any nilpotency class with Euclidean filling functions.

This theorem answers open questions about the filling functions of the Heisenberg groups and the existence of nilpotent groups of large nilpotency class with quadratic 
Dehn functions. Gromov conjectured that the filling functions of the $(2 k+1)$ dimensional Heisenberg group $H^{2 k+1}$ should be Euclidean in dimensions below $k$, super-Euclidean in dimension $k$, and sub-Euclidean in dimensions above $k$ [13], Conj. 5.D.5 (f). Since $J^{1}\left(\mathbb{R}^{k}\right)=H^{2 k+1}$, where $H^{2 k+1}$ is the $2 k+1$-dimensional Heisenberg group, Theorem 1 implies two parts of the conjecture:

Corollary 1.1. For $H^{2 k+1}$ endowed with a left-invariant Riemannian metric,

$$
\mathrm{FV}^{i}(V) \sim \delta^{i-1} \sim V^{\frac{i}{i-1}}
$$

for $i \leq k$, and

$$
\mathrm{FV}^{k+1}(V) \sim \delta^{k} \sim V^{\frac{k+2}{k}}
$$

Furthermore, the jet groups give the first examples of nilpotent groups of arbitrary nilpotency class which have quadratic Dehn function. This is surprising because nilpotent groups with large nilpotency class have highly distorted subgroups; in a class $c$ torsion-free nilpotent group, there are elements $x$ such that $x^{t}$ is distance $\sim t^{1 / c}$ away from the identity in the word metric. As a consequence, the Dehn functions of nilpotent groups with large nilpotency class are often large; for instance, the free $c$-step nilpotent groups have Dehn functions $\delta(n) \sim n^{c+1}$.

The second class of groups we consider are nilpotent groups constructed as quotients by commutators. An important special case is the case of central products of nilpotent groups. These groups generalize the construction used to construct the higher-dimensional Heisenberg groups. Allcock [1] and Ol'shanskii-Sapir [17] studied the higher-dimensional Heisenberg groups with methods that generalize to other central products (see e.g. [16] for one generalization). We will show that bounds on the Dehn functions of many central products follow from these quotient theorems.

In Section 2, we describe some of the objects that we will use in this paper, such as scaling automorphisms of Carnot groups, Lipschitz chains, and Lipschitz triangulations. In Section 3, we describe how one can use triangulations of groups and approximations by simplicial chains to construct fillings of cycles in a nilpotent group. In order to get good bounds on filling inequalities, these triangulations must satisfy certain metric properties, and we construct such triangulations in Section 4. Finally, in Sections 5 and 6, we finish the proof of Theorem 1 by showing upper bounds in the middle dimension and by using cohomological techniques to prove lower bounds on filling inequalities in jet groups.

Note: Much of the work in this paper was done as part of my doctoral thesis at the University of Chicago, and many of the results in this paper first appeared in preprint form as [25] and [26]. The exposition and proofs, however, have been substantially rewritten and improved. The author would like to thank Shmuel Weinberger, Benson Farb, and Stefan Wenger for their help and advice, and to thank the referees for their advice on clarifying and simplifying the exposition. 


\section{Preliminaries}

One inspiration for the study of Dehn functions is the classical isoperimetric problem, which asks for the largest area in the plane which can be enclosed by a loop of a given length. This can be generalized to other spaces and other boundaries; instead of asking about the area enclosed by a loop in the plane, we can ask about the filling volume of a $k$-sphere or $k$-cycle in some other space (i.e., the infimal volume of a $(k+1)$-ball or chain with that sphere or cycle as a boundary). This leads to several possible definitions of higher-order filling invariants, each using a different class of fillings or of boundaries. In this paper, we will mainly use a definition based on Lipschitz chains (i.e., formal sums of Lipschitz maps of simplices), as in [12] and [21]. Other definitions of higher-order filling invariants can be found in [2], [8], [10], [11].

Recall that an integral Lipschitz singular $d$-chain in a space $X$ is a finite linear combination, with integer coefficients, of Lipschitz maps from the Euclidean $d$ dimensional simplex $\Delta^{d}$ to $X$. We will often call this simply a Lipschitz $d$-chain.

We will generally take $X$ to be a Riemannian manifold or simplicial complex, so by Rademacher's Theorem, a Lipschitz map from a simplex to $X$ is differentiable almost everywhere, and one can define the volume of the map as the integral of the magnitude of its jacobian. If $a$ is a Lipschitz $d$-chain, for $d>0$ and $a=\sum_{i} x_{i} \alpha_{i}$ for some maps $\alpha_{i}: \Delta^{d} \rightarrow X$ and some coefficients $x_{i} \in \mathbb{Z}, x_{i} \neq 0$, we define the mass of $a$ as

$$
\operatorname{mass} a:=\sum_{i} x_{i} \operatorname{vol}_{d} \alpha_{i}
$$

The Lipschitz chains form a chain complex, which we denote $C_{*}^{\text {lip }}(X)$. If $g: X \rightarrow Y$ is a Lipschitz map, we can define the pushforward map, $g_{\sharp}: C_{*}^{\text {lip }}(X) \rightarrow C_{*}^{\text {lip }}(Y)$, to be the linear map which sends the simplex $\alpha: \Delta^{d} \rightarrow X$ to the simplex $g \circ \alpha$; this is a map of chain complexes.

If $X$ is a $d$-connected Riemannian manifold or simplicial complex and $a$ is an integral Lipschitz $d$-cycle in $X$, we define the filling volume of $a$ to be

$$
\mathrm{FV}_{X}^{d+1}(a):=\inf \{\operatorname{mass} b \mid \partial b=a\},
$$

where the infimum is taken over the set of $b \in C_{d+1}^{\text {lip }}(X)$ such that $\partial b=a$. We get the $(d+1)$-dimensional filling volume function by taking a supremum over all cycles of a given volume:

$$
\mathrm{FV}_{X}^{d+1}(V):=\sup \left\{\mathrm{FV}_{X}^{d+1}(a) \mid \operatorname{mass} a \leq V\right\},
$$

where the supremum is taken over integral Lipschitz $d$-cycles of mass at most $V$.

The $(d+1)$-dimensional filling volume function is related to the $d$-th order Dehn function, $\delta^{d}$, which measures the volume necessary to extend a map $S^{d} \rightarrow X$ to a map $D^{d+1} \rightarrow X$. If $X$ is a $d$-connected manifold or simplicial complex and 
$f: S^{d} \rightarrow X$ is a Lipschitz map, we define

$$
\delta_{X}^{d}(f):=\inf \left\{\operatorname{vol}_{d} g\left|g: D^{d+1} \rightarrow X, g\right|_{S^{d}}=f\right\}
$$

and

$$
\delta_{X}^{d}(V):=\sup \left\{\delta_{X}^{d}(f) \mid f: S^{d} \rightarrow X, \operatorname{vol}_{d} f \leq V\right\} .
$$

When $d=1$, this is simply called the Dehn function, and it is often written as simply $\delta_{X}(V)$ or even $\delta(V)$ when the space is clear.

The exact relationship between $\delta^{d}$ and $\mathrm{FV}^{d+1}$ depends on $d$. When $d \geq 3$, then $\delta_{X}^{d} \sim \mathrm{FV}_{X}^{d+1}$ for all $d$-connected manifolds or simplicial complexes $X$. When $d=2$, then $\delta_{X}^{d} \lesssim \mathrm{FV}_{X}^{d+1}$. (See [12], App. $2\left(\mathrm{~A}^{\prime}\right)$, or [23] for the upper bound on $\delta^{d}$ and [4], Rem. 2.6 (4), for the lower bound; see also [10], [11].) When $d \leq 2$, the two functions may differ [24], but the bounds found in this paper will hold for both.

A key tool to work with these chains is the Federer-Fleming Deformation Theorem, which states that Lipschitz chains can be approximated by simplicial chains. We will state this theorem in terms of Lipschitz triangulations. A triangulation of a space $X$ consists of a simplicial complex $\tau$ and a homeomorphism $f: \tau \rightarrow X$. We will sometimes refer to the tuple $(\tau, f)$ just as $\tau$, leaving the homeomorphism implicit. We can put a metric on $\tau$ so that each simplex is isometric to the standard Euclidean simplex; if $f$ is a bilipschitz map (i.e., $f$ and $f^{-1}$ are both Lipschitz), then we call $(\tau, f)$ a Lipschitz triangulation. In this paper, all triangulations will be Lipschitz triangulations.

If $(\tau, f: \tau \rightarrow X)$ is a triangulation of $X$, then a simplicial $k$-chain of $\tau$ is a formal sum of $k$-dimensional faces of $\tau$. We can use the push-forward map $f_{\sharp}$ to identify such chains with Lipschitz chains of $X$. By abuse of notation, we call these chains simplicial chains in $X$, and we denote the complex of simplicial chains by $C_{*}(\tau)$. Federer and Fleming showed that Lipschitz chains can be approximated by simplicial chains [9]. The version we state is a slight simplification; it applies only to Lipschitz chains with simplicial boundaries. In this theorem, $P_{\tau}(\alpha)$ will be a simplicial approximation of $\alpha$ and $Q_{\tau}(\alpha)$ will be a Lipschitz chain which interpolates between $\alpha$ and $P_{\tau}(\alpha)$.

Theorem 2 (Deformation Theorem [9], [8]). Let $(\tau, f: \tau \rightarrow X)$ be a triangulation of $X$. There is a constant $c=c(\tau)$ such that if $\alpha \in C_{k}^{\text {lip }}(X)$ is a chain such that $\partial \alpha \in C_{k-1}(\tau)$, then there are $P_{\tau}(\alpha) \in C_{k}(\tau)$ and $Q_{\tau}(\alpha) \in C_{k+1}^{\operatorname{lip}}(X)$ such that

(1) mass $P_{\tau}(\alpha) \leq c \cdot \operatorname{mass}(\alpha)$,

(2) mass $Q_{\tau}(\alpha) \leq c \cdot \operatorname{mass}(\alpha)$, and

(3) $\partial Q_{\tau}(\alpha)=\alpha-P_{\tau}(\alpha)$.

Federer and Fleming originally proved their theorem in the case of Lipschitz currents in $\mathbb{R}^{n}$, so this statement is somewhat different from their original; it is closest to the version proved in [8]. 
We have defined $\delta^{d}$ and $\mathrm{FV}^{d+1}$ as invariants of spaces, but they can in fact be defined as invariants of groups. If $G$ is a group which acts geometrically (that is, cocompactly, properly discontinuously, and by isometries) on a $d$-connected manifold or simplicial complex $X$, then the asymptotic growth rates of $\delta_{X}^{d}(V)$ and $\mathrm{FV}_{X}^{d+1}(V)$ are invariants of $G$. To make this rigorous, we can define a partial ordering on functions $\mathbb{R}^{+} \rightarrow \mathbb{R}^{+}$so that $f \precsim g$ if and only if there is a $c$ such that

$$
f(x) \leq c g(c x+c)+c x+c .
$$

We say $f \sim g$ if and only if $f \precsim g$ and $g \precsim f$. Then if $X_{1}$ and $X_{2}$ are two $d$-connected manifolds or simplicial complexes on which $G$ acts geometrically, then

$$
\delta_{X_{1}}^{d}(V) \sim \delta_{X_{2}}^{d}(V)
$$

and

$$
\mathrm{FV}_{X_{1}}^{d}(V) \sim \mathrm{FV}_{X_{2}}^{d}(V)
$$

and we thus define $\delta_{G}^{d}(V)$ and $\mathrm{FV}_{G}^{d+1}(V)$ to be the $\sim$-equivalence classes of $\delta_{X}^{d}(V)$ and $\mathrm{FV}_{X}^{d+1}(V)$. The fact that this equivalence class is independent of $X$ was first stated by Gromov [13]; Bridson gave a proof in the case that $d=1$ [5], and Alonso, Wang, and Pride proved it for a simplicial version of $\delta^{d}$ when $d \geq 1$ [2]. That proof also applies to a simplicial version of $\mathrm{FV}^{d}$, and one can show that the Lipschitz versions used here are equivalent to simplicial versions using the Deformation Theorem.

In this paper, we focus on finding filling inequalities for Carnot groups, which are nilpotent Lie groups provided with a family of scaling automorphisms. Recall that if $G$ is a simply-connected nilpotent Lie group and $g$ is its Lie algebra, then the lower central series

$$
\mathfrak{g}=\mathfrak{g}_{0} \supset \cdots \supset \mathfrak{g}_{k-1}=\{0\}, \quad \mathfrak{g}_{i+1}=\left[\mathfrak{g}_{i}, \mathfrak{g}\right],
$$

terminates. If $\mathfrak{g}_{k}=\{0\}$ and $\mathfrak{g}_{k-1} \neq\{0\}$, we say that $\mathrm{g}$ has nilpotency class $k$. If there is a decomposition

$$
g=V_{1} \oplus \cdots \oplus V_{k}
$$

such that

$$
\mathfrak{g}_{i}=V_{i+1} \oplus \cdots \oplus V_{k}
$$

and $\left[V_{i}, V_{j}\right] \subset V_{i+j}$ for all $i, j \leq k$, we call it a grading of $\mathrm{g}$. If $\mathrm{g}$ has a grading, we can extend the $V_{i}$ to left-invariant plane fields on $G$ and give $G$ a left-invariant metric such that the $V_{i}$ 's are orthogonal. With this metric, $G$ is called a Carnot group.

If $G$ is a Carnot group, there is a family of automorphisms $s_{t}: G \rightarrow G$ which act on the Lie algebra by $s_{t}(v)=t^{i} v$ for all $v \in V_{i}$. These automorphisms distort vectors in $\mathrm{g}$ by differing amounts. Vectors in $V_{1}$ are distorted the least, and we call these vectors horizontal. If $M$ is a manifold and $f: M \rightarrow G$ is a Lipschitz map, it is differentiable almost everywhere by Rademacher's Theorem. If all of its tangent 
vectors lie in the plane field $V_{1}$, we say that $f$ is horizontal; likewise, if $\alpha \in C^{\operatorname{lip}}(G)$, we say that $\alpha$ is horizontal if it is a sum of horizontal maps. If $\tau$ is a simplicial complex and $f: \tau \rightarrow G$ is a Lipschitz map which is horizontal on every simplex of $\tau$ of dimension at most $k$, we say that $f$ is $k$-horizontal. We then have the following:

Lemma 2.1. If $M$ is a compact $k$-dimensional manifold and $f: \Delta^{k} \rightarrow G$ is horizontal, then $\operatorname{vol}\left(s_{t} \circ f\right)=t^{k} \operatorname{vol} f$ for all $t \geq 0$.

\section{Filling cycles through approximations}

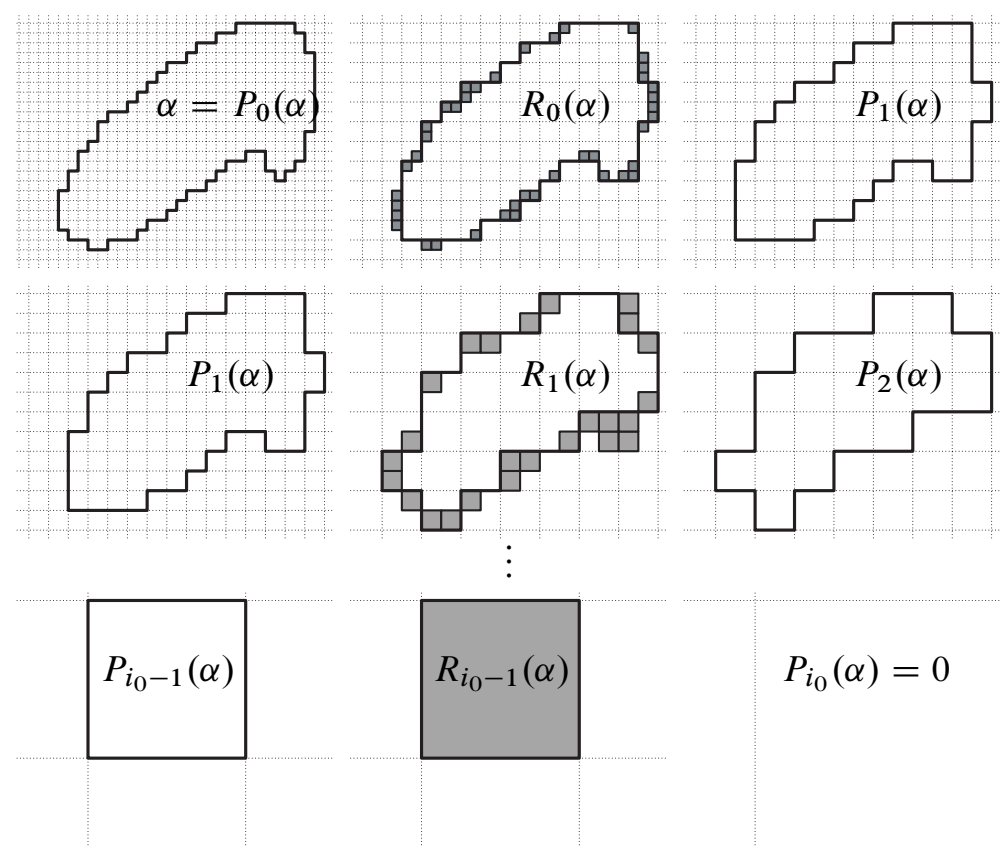

Figure 1. Filling a curve by approximations.

3.1. Sketch of argument. The basic idea behind our techniques is illustrated in Fig. 1, which illustrates a method of bounding the Dehn function of $\mathbb{R}^{2}$. In the figure, $\alpha$ is a curve of length $\ell$ in $\mathbb{R}^{2}$. We approximate $\alpha$ in successively larger grids: $P_{0}(\alpha)$ approximates $\alpha$ in a $1 \times 1$ grid, and $P_{i}(\alpha)$ approximates $\alpha$ in a $2^{i} \times 2^{i}$ grid. Each approximation has length comparable to the length of $\alpha$ when $i$ is small (i.e., $i \leq \log _{2} \ell$ ). When $i>\log _{2} \ell$, then $\alpha$ is smaller than any square in the grid and the curve can be approximated by the zero cycle. We can connect approximations using annuli made of squares, and since $P_{i}(\alpha)$ and $P_{i+1}(\alpha)$ are close together, it takes relatively few such squares; just as $P_{i}(\alpha)$ is made up of $\sim \ell 2^{-i}$ segments of 
length $2^{i}, R_{i}(\alpha)$ is a sum of $\sim \ell 2^{-i}$ squares with side $2^{i}$, and thus has area $\sim \ell 2^{i}$. If $i_{0}$ is such that $2^{i_{0}} \gg \ell$, then $P_{i_{0}}(\alpha)=0$, so we get a filling of $\alpha$ by taking the sum of the $R_{i}(\alpha)$ 's; if we let

$$
\beta=\sum_{i=0}^{i_{0}} R_{i}(\alpha),
$$

then $\partial \beta=\alpha$ and the area of $\beta$ is $\sim \ell^{2}$.

A similar argument can be used to fill higher-dimensional cycles in higher-dimensional Euclidean spaces; if $\alpha$ is a $k$-cycle of mass $V$ in $\mathbb{R}^{n}$, it can be approximated by a sum $P_{i}(\alpha)$ of $\sim V / 2^{i k} k$-cubes of side-length $2^{i}$ by using a $2^{i} \times \cdots \times 2^{i}$ grid in $\mathbb{R}^{n}$. Furthermore, $P_{i}(\alpha)$ and $P_{i+1}(\alpha)$ can be connected by a chain $R_{i}(\alpha)$ consisting of $\sim V / 2^{i k}(k+1)$-cubes of side-length $2^{i}$. As before, if $i_{0}$ is such that $2^{i_{0} k} \gg V$, then $\alpha$ is smaller than any individual cube, so $P_{i_{0}}(\alpha)=0$ and if we let

$$
\beta=\sum_{i=0}^{i_{0}} R_{i}(\alpha),
$$

then we get a filling of $\alpha$ with volume $\sim \ell^{\frac{k+1}{k}}$.

The goal of this paper is to extend this argument to certain nilpotent groups. The key step in doing so is to construct the $P_{i}(\alpha)$ and $R_{i}(\alpha)$; i.e., to construct a sequence of coarser and coarser approximations and then to connect those approximations by chains. In Euclidean space, we use the Federer-Fleming Deformation Theorem to construct $P_{i}$; this theorem allows us to approximate Lipschitz chains by simplicial chains of similar mass, so if we apply it to a $2^{i} \times \cdots \times 2^{i}$ grid, we get an approximation by cubes of side-length $2^{i}$. The connecting chains $R_{i}$ are also produced by simplicial approximation, but the construction is more involved.

The bounds we get on the filling functions of nilpotent groups thus depend on how efficiently we can produce simplicial approximations. The mass of a simplicial approximation is governed by the constant factor $c$ in the Federer-Fleming Deformation Theorem, which depends on the triangulation used. In $\mathbb{R}^{n}$, scaling maps allow us to construct a family of triangulations with differently-sized simplices and the same $c$, but this is not always possible in a nilpotent group. In the rest of this section, we will show that if $G$ is a Carnot group and sufficiently many horizontal maps into $G$ exist, then we can produce efficient simplicial approximations at all scales and use these to find strong bounds on the filling functions of $G$.

Note that we will state and prove our theorems primarily in terms of $\mathrm{FV}^{k+1}$ rather than $\delta^{k}$. At the end of the section, we will outline how to modify these arguments to provide bounds on Dehn functions and their higher-order counterparts.

3.2. Simplicial approximations in Carnot groups. We will construct $P_{i}(\alpha)$ and $R_{i}(\alpha)$ using the Federer-Fleming Deformation Theorem (Theorem 2). This is a tool of geometric measure theory which approximates Lipschitz chains and cycles in $G$ 
by simplicial chains and cycles in a triangulation of $G$. When $G$ is Carnot, we can construct triangulations of $G$ by scaling a single triangulation $\tau$; this gives triangulations with simplices of different scales, and approximations in these triangulations give the $P_{i}$ and $R_{i}$.

One difficulty is that the scaling automorphism may distort these triangulations. The scaling automorphism $s_{t}: \mathbb{R}^{n} \rightarrow \mathbb{R}^{n}$ stretches each direction by a factor of $t$. In a Carnot group, however, the scaling automorphism $s_{t}: G \rightarrow G$ may scale vectors by up to $t^{c}$, where $c$ is the nilpotency class of $G$. Since the $P_{i}$ and $R_{i}$ are made up of scaled simplices, a bad choice of $\tau$ may lead to very large approximations. We avoid this by using $k$-horizontal triangulations. Specifically, we will show that if certain $k$-horizontal maps and triangulations exist, then $P_{i}(\alpha)$ can be constructed so that mass $P_{i}(\alpha) \preceq$ mass $\alpha$ for all $i$, and that this leads to bounds on the filling volume function. (In the next section, we will construct some groups which have such triangulations.)

In the rest of this paper, $G$ will represent a Carnot group and $\Gamma$ a lattice in $G$. The maps $s_{t}: G \rightarrow G, t \geq 0$, will represent the family of scaling automorphisms of $G$.

Let $(\tau, f: \tau \rightarrow G)$ be a triangulation of $G$ and let $P_{\tau}$ and $Q_{\tau}$ be as in Theorem 2. If $f$ is $k$-horizontal and $\alpha$ is a Lipschitz $k$-cycle, then $P_{\tau}(\alpha)$ is a horizontal approximation of $\alpha$. In order to avoid possible issues with constructing horizontal triangulations of $G$, we will construct a slightly different approximation. Let $(\tau, f: \tau \rightarrow G)$ be a triangulation of $G$, with no conditions on the horizontality of $f$. Let $k>0$ be an integer and let $\phi: \tau \rightarrow G$ be a $k$-horizontal Lipschitz map which is a bounded distance from $f$, i.e., there is a $c$ such that $d(f(x), \phi(x))<c$ for all $x \in G$. If $\alpha$ is a Lipschitz $k$-cycle in $G$, then $f_{\#}^{-1}(\alpha)$ is a Lipschitz $k$-cycle in $\tau$, and $P_{\tau}\left(f_{\#}^{-1}(\alpha)\right)$ is a simplicial cycle in $\tau$. By abuse of notation we define $P_{\phi(\tau)}(\alpha)$ by

$$
P_{\phi(\tau)}(\alpha):=\phi_{\sharp}\left[P_{\tau}\left(f_{\#}^{-1}(\alpha)\right)\right] .
$$

This is a sum of images of $k$-simplices of $\tau$, so it is a horizontal cycle.

We will show that the cycle $P_{\phi(\tau)}(\alpha)$ is close to $\alpha$ in the sense that there is a chain $Q_{\phi(\tau)}(\alpha)$ with mass comparable to mass $\alpha$ which interpolates between $\alpha$ and $P_{\phi(\tau)}(\alpha)$.

Lemma 3.1. There is a $c_{Q}$ depending only on $\phi$, $\tau$, and $k$ such that for all $k$-cycles $\alpha$ there is $a(k+1)$-chain $Q_{\phi(\tau)}(\alpha)$ such that

$$
\partial Q_{\phi(\tau)}(\alpha)=P_{\phi(\tau)}(\alpha)-\alpha
$$

and mass $Q_{\phi(\tau)}(\alpha) \leq c_{Q}$ mass $\alpha$.

Proof. Because $\phi \circ f^{-1}$ moves each point of $G$ by a bounded distance, there is a Lipschitz homotopy $h: G \times[0,1] \rightarrow G$ between $\operatorname{id}_{G}$ and $\phi \circ f^{-1}$. Let

$$
Q_{\phi(\tau)}(\alpha):=h_{\sharp}(\alpha \times[0,1])+\phi_{\sharp}\left[Q_{\tau}\left(f_{\sharp}^{-1}(\alpha)\right)\right] .
$$


For the first part of the lemma, note that

$$
\begin{aligned}
\partial Q_{\phi(\tau)}(\alpha) & =h_{\sharp}(\partial(\alpha \times[0,1]))+\phi_{\sharp}\left[\partial Q_{\tau}\left(f_{\sharp}^{-1}(\alpha)\right)\right] . \\
& =\left(\phi_{\sharp}\left(f_{\sharp}^{-1}(\alpha)\right)-\alpha\right)+\phi_{\sharp}\left[P_{\tau}\left(f_{\sharp}^{-1}(\alpha)\right)-f_{\sharp}^{-1}(\alpha)\right] \\
& =\phi_{\sharp}\left(f_{\sharp}^{-1}(\alpha)\right)-\alpha+\phi_{\sharp}\left[P_{\tau}\left(f_{\sharp}^{-1}(\alpha)\right)\right]-\phi_{\sharp}\left(f_{\sharp}^{-1}(\alpha)\right) \\
& =P_{\phi(\tau)}(\alpha)-\alpha .
\end{aligned}
$$

By Theorem 2, there is a $c$ such that

$$
\operatorname{mass} Q_{\tau}\left(f_{\sharp}^{-1}(\alpha)\right) \leq c \text { mass } f_{\sharp}^{-1}(\alpha) \text {. }
$$

If we let

$$
c_{Q}:=\operatorname{Lip}(h)^{k+1}+\operatorname{Lip}\left(f^{-1}\right)^{k} \operatorname{Lip}(\phi)^{k+1} c,
$$

it is then straightforward to check the second part of the lemma.

We can compose $P_{\phi(\tau)}$ with scaling automorphisms to produce a sequence of approximations. To avoid cumbersome subscripts, we will abuse notation by writing $s_{t}(\alpha)$ instead of $\left(s_{t}\right)_{\sharp}(\alpha)$ when the intention is clear. We define

$$
P_{i}(\alpha)=s_{2^{i}}\left(P_{\phi(\tau)}\left(s_{2^{-i}}(\alpha)\right)\right)
$$

this is a horizontal approximation of $\alpha$ with simplices of diameter $\sim 2^{i}$.

Lemma 3.2. There is a $c_{P}$ depending only on $\phi$ and $\tau$ such that for all $i \geq 0$ and for all $k$-cycles $\alpha$, we have mass $P_{i}(\alpha) \leq c_{P}$ mass $\alpha$.

Proof. Note that by the choice of the metric on $G$, for any $k$-chain $\sigma$ and any $0 \leq$ $t \leq 1$,

$$
\operatorname{mass} s_{t}(\sigma) \leq t^{k} \operatorname{mass} \sigma
$$

and that for any horizontal $k$-chain $\sigma$ (in particular, when $\sigma=P_{\phi(\tau)}\left(s_{2-i}(\alpha)\right)$ ) and any $t>0$,

$$
\operatorname{mass} s_{t}(\sigma)=t^{k} \operatorname{mass} \sigma \text {. }
$$

By Theorem 2, there is a $c$ such that

$$
\operatorname{mass} P_{\tau}(\sigma) \leq c \text { mass } \sigma
$$

for all $k$-chains $\sigma$.

If we let $c_{P}:=\operatorname{Lip}\left(f^{-1}\right)^{k} \operatorname{Lip}(\phi)^{k} c$, then

$$
\operatorname{mass} P_{i}(\alpha) \leq 2^{i k} \operatorname{Lip}\left(f^{-1}\right)^{k} \operatorname{Lip}(\phi)^{k} c 2^{-i k} \operatorname{mass} \alpha \leq c_{P} \operatorname{mass} \alpha,
$$

as desired. 
Next, we construct simplicial chains interpolating between two different approximations of a cycle. The basic idea is that if $\left(\tau_{0}, f_{0}: \tau_{0} \rightarrow G\right)$ and $\left(\tau_{1}, f_{1}: \tau_{1} \rightarrow G\right)$ are two triangulations of $G$, we can connect approximations in $\tau_{0}$ and $\tau_{1}$ using a triangulation of $G \times[0,1]$ which interpolates between $\tau_{0}$ and $\tau_{1}$.

Let $(\eta, g: \eta \rightarrow G \times[0,1])$ be a triangulation of $G \times[0,1]$. For $i=0,1$, suppose that $g^{-1}(G \times\{i\})$ is a subcomplex of $\eta$ which is isomorphic to $\tau_{i}$ under an isomorphism $\iota_{i}: \tau_{i} \cong g^{-1}(G \times\{i\})$ such that $g \circ \iota_{i}=f_{i}$. We say that $\eta$ restricts to $\tau_{i}$ on $G \times\{i\}$. Let $\psi: \eta \rightarrow G$ be a $(k+1)$-horizontal map and let $\phi_{i}: \tau_{i} \rightarrow G$ be defined by $\phi_{i}=\psi \circ \iota_{i}$ for $i=0,1$. We will construct a horizontal $(k+1)$-chain interpolating between $P_{\phi_{0}\left(\tau_{0}\right)}(\alpha)$ and $P_{\phi_{1}\left(\tau_{1}\right)}(\alpha)$.

Lemma 3.3. There is a $c_{R}$ depending only on $\psi$ and $\eta$ such that for all $i \geq 0$ and for all $k$-cycles $\alpha$, there is a $(k+1)$-chain $R_{\psi(\eta)}(\alpha)$ such that

$$
\partial R_{\psi(\eta)}(\alpha)=P_{\phi_{1}\left(\tau_{1}\right)}(\alpha)-P_{\phi_{0}\left(\tau_{0}\right)}(\alpha)
$$

and

$$
\operatorname{mass} R_{\psi(\eta)}(\alpha) \leq c_{R} \operatorname{mass} \alpha .
$$

Furthermore, this chain is the image under $\psi_{\sharp}$ of a simplicial chain in $\eta$, so it is horizontal.

Proof. Let

$$
X(\alpha):=Q_{\tau_{1}}\left[\left(f_{1}^{-1}\right)_{\sharp}(\alpha)\right]+g_{\sharp}^{-1}(\alpha \times[0,1])-Q_{\tau_{0}}\left[\left(f_{0}^{-1}\right)_{\sharp}(\alpha)\right],
$$

and note that

$$
\partial X(\alpha)=P_{\tau_{1}}\left[\left(f_{1}^{-1}\right)_{\sharp}(\alpha)\right]-P_{\tau_{0}}\left[\left(f_{0}^{-1}\right)_{\sharp}(\alpha)\right] .
$$

Define

$$
R_{\psi(\eta)}(\alpha):=\psi_{\sharp}\left(P_{\eta}(X(\alpha))\right) .
$$

This is the image of a simplicial chain, and

$$
\partial R_{\psi(\eta)}(\alpha)=\psi_{\sharp}(\partial X(\alpha))=P_{\phi_{1}\left(\tau_{1}\right)}(\alpha)-P_{\phi_{0}\left(\tau_{0}\right)}(\alpha),
$$

as desired. For the bound on the mass of $R_{\psi(\eta)}(\alpha)$, note that by Theorem 2, there is a $c$ such that

$$
\operatorname{mass} P_{\tau}(\sigma) \leq c \operatorname{mass} \sigma
$$

and

$$
\text { mass } Q_{\tau}(\sigma) \leq c \text { mass } \sigma
$$

for all $k$-chains or $(k+1)$-chains $\sigma$. Thus

$$
\operatorname{mass} X(\alpha) \leq\left[2 c\left(\operatorname{Lip~}^{-1}\right)^{k}+\left(\operatorname{Lip} g^{-1}\right)^{k+1}\right] \operatorname{mass} \alpha .
$$


If we let

$$
c_{R}:=c\left[2 c\left(\operatorname{Lip} g^{-1}\right)^{k}+\left(\operatorname{Lip} g^{-1}\right)^{k+1}\right] \operatorname{Lip}(\psi)^{k+1},
$$

then

$$
\operatorname{mass} R_{\psi(\eta)}(\alpha) \leq c_{R} \operatorname{mass} \alpha .
$$

In this case, we would like to connect $P_{0}(\alpha)=P_{\phi(\tau)}(\alpha)$ and

$$
P_{1}(\alpha)=s_{2}\left(P_{\phi(\tau)}\left(s_{2^{-1}}(\alpha)\right)\right) .
$$

Let $\left(\tau_{0}, f_{0}: \tau_{0} \rightarrow G\right)=(\tau, f)$ and $\phi_{0}=\phi$, and define $\left(\tau_{1}, f_{1}: \tau_{1} \rightarrow G\right)$ by letting $\tau_{1}=\tau$ and $f_{1}=s_{2} \circ f$. If we define $\phi_{1}=s_{2} \circ \phi$, then we have $P_{1}(\alpha)=P_{\phi_{1}\left(\tau_{1}\right)}(\alpha)$. We will define $R_{0}(\alpha)$ as $R_{\psi(\eta)}(\alpha)$ for an appropriate $\psi$ and $\eta$ and obtain $R_{i}$ by conjugating $R_{0}$ by $s_{2^{i}}$.

Lemma 3.4. Let $k>0$. Let $(\tau, f: \tau \rightarrow G)$ be a triangulation and let $\phi: \tau \rightarrow G$ be a $(k+1)$-horizontal map which is a bounded distance from $f$. Define $\tau_{0}, \phi_{0}, \tau_{1}$ and $\phi_{1}$ as above.

Let $(\eta, g: \eta \rightarrow G \times[0,1])$ be a triangulation of $G \times[0,1]$ which restricts to $\tau_{i}$ on $G \times\{i\}, i=0,1$. Let $\iota_{i}: \tau_{i} \cong g^{-1}(G \times\{i\})$ be the implied isomorphism. Let $\psi: \eta \rightarrow G$ be a $(k+1)$-horizontal map which extends the $\phi_{i}\left(i . e ., \phi_{i}=\psi \circ \iota_{i}, i=\right.$ $0,1)$. If we define

$$
R_{i}(\alpha):=s_{2^{i}}\left(R_{\psi(\eta)}\left(s_{2^{-i}}(\alpha)\right)\right),
$$

then for all $i \geq 0$ and for all $k$-cycles $\alpha$ we have

$$
\partial R_{i}(\alpha)=P_{i+1}(\alpha)-P_{i}(\alpha)
$$

and

$$
\operatorname{mass} R_{i}(\alpha) \leq c_{R} 2^{i} \operatorname{mass} \alpha,
$$

where $c_{R}$ is the constant from Lemma 3.3 corresponding to $\psi$ and $\eta$.

Proof. It follows from Lemma 3.3 that

$$
\begin{aligned}
\partial R_{i}(\alpha) & =s_{2^{i}}\left[P_{\phi_{1}\left(\tau_{1}\right)}\left(s_{2^{-i}}(\alpha)\right)-P_{\phi_{0}\left(\tau_{0}\right)}\left(s_{2^{-i}}(\alpha)\right)\right] \\
& =\left(s_{2^{i}} \circ P_{\phi_{1}\left(\tau_{1}\right)} \circ s_{2^{-i}}\right)(\alpha)-\left(s_{2^{i}} \circ P_{\phi_{0}\left(\tau_{0}\right)} \circ s_{2^{-i}}\right)(\alpha) .
\end{aligned}
$$

Since

$$
P_{\phi_{1}\left(\tau_{1}\right)}(\alpha)=\left(s_{2} \circ P_{\phi_{0}\left(\tau_{0}\right)} \circ s_{2^{-1}}\right)(\alpha),
$$

we have $\partial R_{i}(\alpha)=P_{i+1}(\alpha)-P_{i}(\alpha)$ as desired.

Next we bound the mass of $R_{i}(\alpha)$. Recall that mass $R_{0}(\alpha) \leq c_{R}$ mass $\alpha$ for all $\alpha$. Then

$$
\operatorname{mass} R_{i}(\alpha)=\operatorname{mass} s_{2^{i}}\left(R_{i}\left(s_{2^{-i}}(\alpha)\right)\right) \text {. }
$$

Since $R_{i}\left(s_{2^{-i}}(\alpha)\right)$ is a horizontal $(k+1)$-cycle, we have

$$
\operatorname{mass} R_{i}(\alpha) \leq 2^{(k+1) i} c_{R} 2^{-i k} \operatorname{mass} \alpha=c_{R} 2^{i} \operatorname{mass} \alpha,
$$

as desired. 
When such an $R_{i}$ exists, we can use it to prove filling inequalities.

Theorem 3. Let $k,(\tau, f), \phi,(\eta, g)$, and $\psi$ satisfy the hypotheses of Lemma 3.4. Then

$$
\mathrm{FV}_{G}^{k+1}(V) \preceq V^{\frac{k+1}{k}} .
$$

Proof. It suffices to show that there is a $c$ such that if $\alpha$ is a $k$-cycle with sufficiently large volume, then there is a chain $\beta$ such that $\partial \beta=\alpha$ and

$$
\operatorname{mass} \beta \leq c(\operatorname{mass} \alpha)^{\frac{k+1}{k}} \text {. }
$$

First, we claim that when $i$ is large, then $P_{i}(\alpha)=0$. Let

$$
c^{\prime}=c_{\tau} \operatorname{Lip}\left(f^{-1}\right)^{k}\left(\operatorname{vol} \Delta^{k}\right)^{-1},
$$

where $c_{\tau}$ is the constant from Theorem 2 and $\Delta^{k}$ is the standard Euclidean $k$-simplex. Let $i_{0}$ be the integer such that

$$
2^{\left(i_{0}-1\right) k} \leq c^{\prime} \operatorname{mass} \alpha<2^{i_{0} k},
$$

and suppose that $i \geq i_{0}$.

Let $X=\left(P_{\tau} \circ \bar{f}_{\sharp}^{-1} \circ S_{2}-i\right)(\alpha)$, so that $P_{i}(\alpha)=s_{2^{i}}\left(\phi_{\sharp}(X)\right)$. We claim that $X=0$. Since $X$ is an integral simplicial $k$-cycle, it suffices to show that mass $X<\operatorname{vol} \Delta^{k}$. But, by Theorem 2, we have

$$
\operatorname{mass} X \leq c_{\tau} \operatorname{Lip}\left(f^{-1}\right)^{k} 2^{-k i} \operatorname{mass} \alpha<\operatorname{vol} \Delta^{k},
$$

so $X=0$ and thus $P_{i}(\alpha)=0$.

We claim that

$$
\beta:=-\left(Q_{\phi(\tau)}(\alpha)+\sum_{i=0}^{i_{0}-1} R_{i}(\alpha)\right)
$$

is a filling of $\alpha$ with mass $\preceq(\operatorname{mass} \alpha)^{(k+1) / k}$. First, note that

$$
\partial \beta=\alpha-P_{i_{0}}(\alpha)=\alpha .
$$

Furthermore, by Lemmas 3.4 and 3.1, we have

$$
\begin{aligned}
\operatorname{mass} \beta & \leq c_{Q} \operatorname{mass} \alpha+\sum_{i=0}^{i_{0}-1} c_{R} 2^{i} \operatorname{mass} \alpha \\
& \leq\left(c_{Q}+c_{R} 2^{i_{0}}\right) \operatorname{mass} \alpha \\
& \leq\left(c_{Q}+2 c_{R}\left(c^{\prime} \operatorname{mass} \alpha\right)^{1 / k}\right) \operatorname{mass} \alpha .
\end{aligned}
$$

If mass $\alpha$ is sufficiently large, then we have

$$
\operatorname{mass} \beta \leq 4 c_{R}\left(c^{\prime} \operatorname{mass} \alpha\right)^{\frac{k+1}{k}},
$$

as desired. 
So we can get filling inequalities by constructing triangulations and $k$-horizontal maps. In the next section, we will describe two ways of constructing such triangulations.

3.3. Homotopic filling bounds. In this section, we give a sketch of how to adapt the above arguments to produce fillings of spheres by balls rather than fillings of chains by cycles. Note that when $d \geq 2$, an upper bound on $\mathrm{FV}^{d+1}$ implies a bound on $\delta^{d}$; Gromov [12], App. $2\left(\mathrm{~A}^{\prime}\right)$, and White [23] showed that $\delta^{d} \preceq \mathrm{FV}^{d+1}$ (with equality when $d \geq 3$ ).

The main change to produce homotopic fillings rather than homological fillings is a homotopic version of the Deformation Theorem. To state this, we recall the admissible maps used by Brady, Bridson, Forester, and Shankar [4]. If $M$ is a compact $k$-dimensional manifold and $\tau$ is a simplicial complex, a map $\alpha: M \rightarrow \tau$ is admissible if the image of $\alpha$ lies in the $k$-skeleton $\tau^{(k)}$ of $\tau$, and if $\alpha^{-1}\left(\tau^{(k)}-\tau^{(k-1)}\right)$ is a disjoint union of open $k$-balls, each mapped homeomorphically onto a $k$-cell of $\tau$. If $(\tau, f: \tau \rightarrow N)$ is a triangulation of $N$, we say that $\alpha: M \rightarrow N$ is $\tau$-admissible if and only if $f^{-1} \circ \alpha$ is an admissible map to $\tau$. One can then prove the following:

Theorem 4 (Homotopic Deformation Theorem). Let $(\tau, f: \tau \rightarrow X)$ be a triangulation of $X$ and let $M$ be a compact $k$-manifold.

There is a constant $c=c(\tau)$ such that if $\alpha: M \rightarrow X$ is a Lipschitz map and $\left.\alpha\right|_{\partial M}$ is $\tau$-admissible, then there is a $\tau$-admissible map $P_{\tau}^{\prime}(\alpha): M \rightarrow X$ and a Lipschitz homotopy $Q_{\tau}^{\prime}(\alpha): M \times[0,1] \rightarrow X$ such that

(1) $\operatorname{vol} P_{\tau}^{\prime}(\alpha) \leq c \cdot \operatorname{vol} \alpha$,

(2) $\operatorname{vol} Q_{\tau}^{\prime}(\alpha) \leq c \cdot \operatorname{vol} \alpha$, and

(3) $Q_{\tau}^{\prime}(\alpha)$ is a homotopy between $\alpha$ and $P_{\tau}^{\prime}(\alpha)$ which is constant on $\partial M$.

This can be used to prove:

Theorem 5. Let $k,(\tau, f), \phi,(\eta, g)$, and $\psi$ satisfy the hypotheses of Lemma 3.4. Then

$$
\delta_{G}^{k}(V) \preceq V^{\frac{k+1}{k}} .
$$

Sketch of proof. If $\alpha: S^{k} \rightarrow X$, we can use the Homotopic Deformation Theorem to construct $\left(\tau, s_{2^{i}} \circ f\right)$-admissible maps $P_{i}^{\prime}(\alpha): S^{k} \rightarrow X$ approximating $\alpha$ at different scales and horizontal homotopies $R_{i}^{\prime}(\alpha)$ which connect the $P_{i}^{\prime}(\alpha)$ 's. When $k,(\tau, f)$, $\phi,(\eta, g)$, and $\psi$ satisfy the hypotheses of Lemma 3.4, these maps and homotopies satisfy the same bounds on their volumes as their homological counterparts.

We construct a disc filling $\alpha$ by connecting the $R_{i}^{\prime}(\alpha)$ end-to-end for $i=0, \ldots, i_{0}$; this gives us a homotopy between $P_{0}^{\prime}(\alpha)=P_{\tau}^{\prime}(\alpha)$ and $P_{i_{0}}^{\prime}(\alpha)$. The map $P_{0}^{\prime}(\alpha)$ is homotopic to $\alpha$ by the homotopy $Q_{\tau}^{\prime}(\alpha)$. Since $P_{i_{0}}^{\prime}(\alpha)$ is $\left(\tau, s_{2^{i}} \circ f\right)$-admissible, the map $\alpha^{\prime}=f^{-1} \circ s_{2^{-i}} \circ P_{i_{0}}^{\prime}(\alpha)$ is admissible, but if $i_{0}$ is sufficiently large, 
$\operatorname{vol} \alpha^{\prime}<\operatorname{vol} \Delta^{k}$. This implies that the image of $\alpha^{\prime}$ lies entirely in the $(k-1)$-skeleton of $\tau$, so $\operatorname{vol}^{k} P_{i_{0}}^{\prime}(\alpha)=0$. There is thus a homotopy contracting $P_{i_{0}}^{\prime}(\alpha)$ to a point which has no $(k+1)$-volume. Combining all of these homotopies, we get a homotopy from $\alpha$ to a point whose volume is $\preceq(\operatorname{vol} \alpha)^{(k+1) / k}$. This homotopy is the required disc filling $\alpha$.

\section{Constructing horizontal triangulations}

Theorems 3 and 5 show that when certain triangulations and $k$-horizontal maps exist, then $G$ satisfies Euclidean filling inequalities. We will give two families of examples of groups with such maps and triangulations, one using combinatorial group theory and one using geometry. The combinatorial example gives a way to prove quadratic bounds on the Dehn functions of central products; these groups include the higherdimensional Heisenberg groups and have been studied by Ol'shanskii and Sapir [17], Allcock [1], and Magnani [16]. The geometric example shows that jet groups have enough horizontal submanifolds to construct the required maps and triangulations, and that consequently, their filling functions satisfy Euclidean bounds.

4.1. Central products. If $G$ is a 2-step nilpotent group with center $Z$, we define the central product $G \times{ }_{Z} G$ to be the quotient $G \times G / \sim$, where $\sim$ is the relation which identifies the centers of the two copies of $G$ (i.e., the relation $(z, 1) \sim(1, z)$ for all $z \in Z$ ). Likewise, we define the $n$-fold central product to be the quotient

$$
G^{\times} Z^{n}=G^{n} / \sim
$$

where $\sim$ is the relation

$$
(z, 1,1, \ldots) \sim(1, z, 1, \ldots) \sim(1,1, z, \ldots) \sim \ldots \text { for all } z \in Z
$$

which identifies the centers of all the copies of $Z$. These groups include the higherdimensional Heisenberg groups, which are given by $H_{2 n+1}=\left(H_{3}\right)^{\times} Z^{n}$. Central products also appear as cusp groups in lattices in rank 1 symmetric spaces.

In this section, we will prove Dehn function bounds for such groups. First, we will show that central products of free nilpotent groups have quadratic Dehn functions. A central product of any 2-step nilpotent group is a quotient of a central product of a free nilpotent group. We will show that any such quotient has a Dehn functions which grows at most as fast as $n^{2} \log n$ and that many such quotients have quadratic Dehn functions.

Consider the free 2-step nilpotent group on $r$ generators, which is given by the presentation:

$$
\left.\Lambda_{r}=\left\langle g_{1}, \ldots, g_{r}\right|\left[g_{i},\left[g_{j}, g_{k}\right]\right]=0 \text { for } 1 \leq i, j, k \leq r .\right\rangle
$$


Its abelianization is $\mathbb{Z}^{r}$, generated by the $g_{i}$, and its center is isomorphic to $\mathbb{Z}\left(\begin{array}{l}r \\ 2\end{array}\right)$, generated by elements of the form $\left[g_{i}, g_{j}\right], 1 \leq i<j \leq r$. Define $\Lambda_{r, n}=\left(\Lambda_{r}\right)^{\times Z^{n}}$. We claim:

Proposition 4.1. $\Lambda_{r, n}$ has a quadratic Dehn function when $n \geq 2$.

This proposition was first stated without proof by Ol'shanskii and Sapir [17]. It will follow from applying Theorem 5 with $k=1$. We will show that $\Lambda_{r, n}$ satisfies the conditions of the theorem by finding a presentation of $\Lambda_{r, n}$ and then using that presentation to construct triangulations and horizontal maps.

These groups are lattices in nilpotent Lie groups: $\Lambda_{r}$ is a lattice in the free 2-step nilpotent Lie group of rank $r$, which we call $F_{r}$, and $\Lambda_{r, n}$ is a lattice in $F_{r, n}:=\left(F_{r}\right)^{\times Z^{n}}$. Let $\mathfrak{f}_{r}$ be the Lie algebra of $F_{r}$ and let $v_{i}=\log g_{i} \in \mathfrak{f}_{r}$. If we define generators of $\mathfrak{f}_{r}$ by $v_{i}=\log g_{i} \in \mathfrak{f}_{r}$, then $\mathfrak{f}_{r}$ has a grading

$$
f_{r}=V_{1} \oplus V_{2}=\left\langle v_{1}, \ldots, v_{r}\right\rangle \oplus\left\langle\left[v_{i}, v_{j}\right] \text { for all } 1 \leq i<j \leq r\right\rangle .
$$

If $g_{i j}$ is the $i$ th generator of the $j$ th factor in the central product $F_{r, n}$, and $f_{r, n}$ is its Lie algebra, we can likewise define a grading

$$
\mathrm{f}_{r, n}=V_{1}^{n} \oplus V_{2}=\left\langle v_{11}, \ldots, v_{r n}\right\rangle \oplus\left\langle\left[v_{i 1}, v_{j 1}\right] \text { for all } 1 \leq i<j \leq r\right\rangle
$$

where $v_{i j}=\log g_{i j}$.

For ease of notation, we will start by considering $\Lambda_{r, 2}$. Let $g_{1}, \ldots, g_{r} \in \Lambda_{r} \times \Lambda_{r}$ be the generators of the first factor and let $h_{1}, \ldots, h_{r} \in \Lambda_{r} \times \Lambda_{r}$ be the generators of the second. Then $\Lambda_{r, 2}$ is given by the presentation:

$$
\begin{array}{r}
\Lambda_{r, 2}=\left\langle g_{1}, \ldots, g_{r}, h_{1}, \ldots, h_{r}\right|\left[g_{i},\left[g_{j}, g_{k}\right]\right]=0 \text { for } 1 \leq i, j, k \leq r, \\
{\left[h_{i},\left[h_{j}, h_{k}\right]\right]=0 \text { for } 1 \leq i, j, k \leq r,} \\
{\left[g_{i}, h_{j}\right]=0 \text { for } 1 \leq i, j \leq r,} \\
\left.\left[g_{i}, g_{j}\right]\left[h_{j}, h_{i}\right]=0 \text { for } 1 \leq i, j \leq r\right\rangle .
\end{array}
$$

We claim:

Lemma 4.2. $\Lambda_{r, 2}$ can be presented as:

$$
\begin{array}{r}
\Lambda_{r, 2}=\left\langle g_{1}, \ldots, g_{r}, h_{1}, \ldots, h_{r}\right|\left[g_{i}, h_{j}\right]=0 \text { for } 1 \leq i, j \leq r, \\
\left.\left[g_{i} h_{j}, g_{j} h_{i}\right]=0 \text { for } 1 \leq i, j \leq r\right\rangle .
\end{array}
$$

Proof. We need to show that the relation $\left[g_{i} h_{j}, g_{j} h_{i}\right]=0$ holds in $\Lambda_{r, 2}$ for all $i$ and $j$, and we need to show that relations (1), (2), and (4) can be deduced from (5) and (6) (since (3) and (5) are the same).

First, we reduce $\left[g_{i} h_{j}, g_{j} h_{i}\right]$ to the empty word $\varepsilon$ by using (1)-(4):

$$
\begin{aligned}
{\left[g_{i} h_{j}, g_{j} h_{i}\right] } & \rightarrow\left[g_{i}, g_{j}\right]\left[h_{j}, h_{i}\right] \quad \text { by }(3) \\
& \rightarrow \varepsilon \text { by }(4) .
\end{aligned}
$$


In the first step, we shuffled all the $g_{i}$ 's and $g_{j}$ 's in $\left[g_{i} h_{j}, g_{j} h_{i}\right]$ to the beginning using the fact that $g$ 's and $h$ 's commute.

Now we deduce (1), (2), and (4) from (5) and (6). First, note that (4) follows from (5) and (6) by the reverse of the argument above:

$$
\left[g_{i}, g_{j}\right]\left[h_{j}, h_{i}\right] \rightarrow\left[g_{i} h_{j}, g_{j} h_{i}\right] \rightarrow \varepsilon .
$$

Next, we can reduce (1) as follows:

$$
\begin{aligned}
{\left[g_{i},\left[g_{j}, g_{k}\right]\right] } & \rightarrow\left[g_{i},\left[h_{j}, h_{k}\right]\right] \quad \text { by }(4) \\
& \rightarrow \varepsilon \quad \text { by }(5) .
\end{aligned}
$$

Here we reduced $\left[g_{i},\left[h_{j}, h_{k}\right]\right]$ to the trivial word by using the fact that $g_{i}$ commutes with each letter of $\left[h_{j}, h_{k}\right]$. The same procedure can be used to deduce (2).

Larger central products have a similar presentation; here, $g_{i j}$ represents the $i$ th generator of the $j$ th factor of $\Lambda_{r}^{n}$ :

Lemma 4.3. $\Lambda_{r, n}$ can be presented as:

$$
\begin{array}{r}
\Lambda_{r, n}=\left\langle g_{i j}, i=1, \ldots, r, j=1, \ldots, n\right|\left[g_{i j}, g_{k l}\right]=0 \text { for all } j \neq l, \\
\left.\left[g_{i j} g_{k l}, g_{k j} g_{i l}\right]=0 \text { for all } j \neq l\right\rangle .
\end{array}
$$

We omit the proof, which follows the outline of Lemma 4.2.

Recall that if $\Gamma$ is a group with a finite presentation

$$
\Gamma=\left\langle x_{1}, \ldots, x_{d} \mid r_{1}, \ldots, r_{s}\right\rangle,
$$

then its Cayley complex $X_{\Gamma}$ is a simply-connected 2-complex on which $\Gamma$ acts geometrically (that is, properly discontinuously, cocompactly, and by isometries). The 1-skeleton of $X_{\Gamma}$ is the Cayley graph of $\Gamma$ with respect to the $x_{i}$. At each vertex of the Cayley graph, there is a loop corresponding to each relator $r_{i}$, and we obtain $X_{\Gamma}$ by gluing a 2-cell to each such loop. Since we started with a presentation of $\Gamma$, this procedure results in a simply-connected complex.

The advantage of the presentation in (7) is that there is a horizontal map from its Cayley complex $X:=X_{\Lambda_{r, n}}$ to $F_{r, n}$, which we will denote $h: X \rightarrow F_{r, n}$. Vertices of $X$ correspond to elements of $\Lambda_{r, n}$, so we map each vertex to the corresponding element of $F_{r, n}$. Each edge $e$ of $X$ connects $g$ and $g g_{i j}^{ \pm 1}$ for some $g \in \Lambda_{r, n}$ and some $i$ and $j$. Since $g_{i j}=\exp v_{i j}$ for all $i, j$, the points $g$ and $g g_{i j}^{ \pm 1}$ can be connected by a horizontal segment of the form $t \mapsto g \exp \pm t v_{i j}, 0 \leq t \leq 1$; we define $h(e)$ to be this segment. Then if $w=w_{1} \ldots w_{p}$ is a word, it corresponds to an edge path in $X$ and its image under $h$ is a horizontal curve $\gamma_{w}$ in $G$ which connects the points $0, w_{1}$, $w_{1} w_{2}, \ldots, w_{1} \ldots w_{p}$. Each relation in (7) then corresponds to a horizontal closed curve $\gamma_{w}$. We complete the definition of $h$ by filling these curves with horizontal discs. 
First, we consider $w=\left[g_{i j}, g_{k l}\right]$. The curve $\gamma_{w}$ lies in the 2-parameter subgroup $\exp \left\langle v_{i j}, v_{k l}\right\rangle$, and we can fill it with a disc of the form $\exp \left(s v_{i j}+t v_{k l}\right), 0 \leq s, t \leq 1$. Next we consider $w=\left[g_{i j} g_{k l}, g_{k j} g_{i l}\right]$. Here, the disc is a little more complicated and is shown in Figure 2.

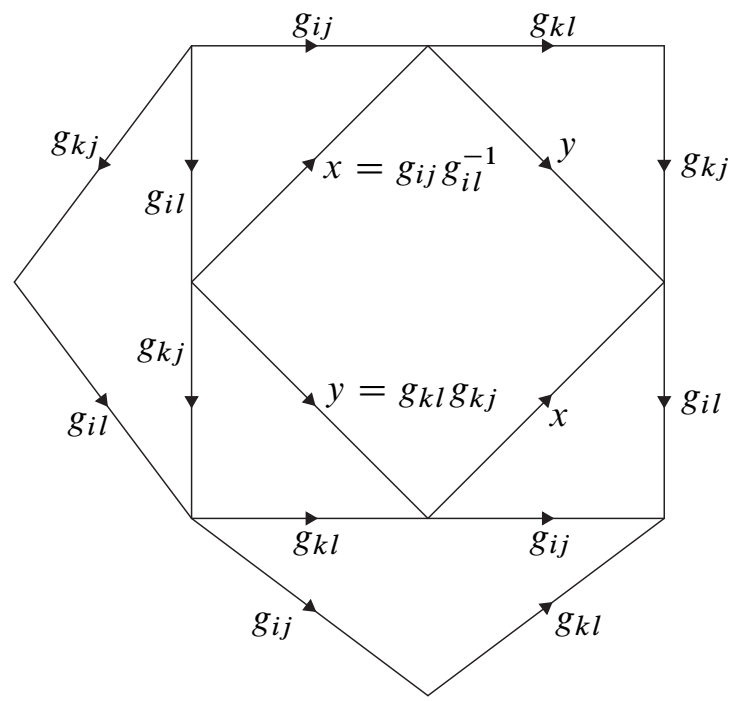

Figure 2. A disc filling $\gamma_{w}$ for $w=\left[g_{i j} g_{k l}, g_{k j} g_{i l}\right]$.

The disc is made up of three quadrilaterals and four triangles. Each edge in the disc is a segment of a translate of a horizontal 1-parameter subgroup, and each face lies in a translate of a 2-dimensional horizontal subgroup of $F_{r, n}$. The quadrilateral on the left lies in a translate of the subgroup with Lie algebra $\left\langle v_{i l}, v_{k j}\right\rangle$, the one on the bottom lies in a translate of $\exp \left\langle v_{k l}, v_{i j}\right\rangle$, and the one in the center lies in a translate of $\exp \left\langle v_{i j}-v_{i l}, v_{k l}+v_{k j}\right\rangle$. Likewise, each triangle lies in a translate of either $\exp \left\langle v_{i j}, v_{i l}\right\rangle$ or $\exp \left\langle v_{k j}, v_{k l}\right\rangle$. All of these subgroups are horizontal submanifolds of $F_{r, n}$, so the disc is horizontal.

As a consequence, we conclude the following:

Lemma 4.4. If $w$ is a word in $\Lambda_{r, n}$ which represents the identity, then there is a horizontal disc filling $\gamma_{w}$ in $F_{r, n}$.

Proof. Since $w$ represents the identity, it corresponds to a closed edge path in $X$, and since $X$ is simply connected, this path can be filled by a disc in $X$. The image of this disc in $F_{r, n}$ is a disc filling $\gamma_{w}$.

We can use this lemma to construct triangulations and maps satisfying the hypotheses of Theorem 5 with $k=1$. To apply the theorem to a group $G$, we need a triangulation $\eta$ of $G \times[0,1]$ which restricts to triangulations $\tau_{0}$ and $\tau_{1}$ on $G \times\{0\}$ 
and $G \times\{1\}$ respectively, where $\tau_{1}$ is a scaling of $\tau_{0}$. In the following lemma, we show that if $G$ contains a lattice $\Gamma$ such that $\Gamma \subset s_{2}(\Gamma)$, then $\tau_{0}$ can be chosen to be $\Gamma$-equivariant and $\eta$ can be chosen to be $s_{2}(\Gamma)$-equivariant.

Lemma 4.5. Let $\Gamma$ be a lattice in a Carnot group $G$ such that $s_{2}(\Gamma) \subset \Gamma$ and let $(\tau, f: \tau \rightarrow G)$ be a $\Gamma$-equivariant triangulation. Let $\left(\tau_{i}, f_{i}: \tau_{i} \rightarrow G\right), i=0,1$ be triangulations such that $\tau_{0}=\tau_{1}=\tau, f_{0}=f$, and $f_{1}=s_{2} \circ f_{0}$. Then there is an $s_{2}(\Gamma)$-equivariant triangulation $(\eta, g: \eta \rightarrow G \times[0,1])$ of $G \times[0,1]$ which restricts to $\tau_{i}$ on $G \times\{i\}$ for $i=0,1$.

Proof. First, we construct $s_{2}(\Gamma)$-actions on $\tau_{0}$ and $\tau_{1}$ which make them equivariant triangulations. Let $\rho(g): \tau \rightarrow \tau, g \in \Gamma$ be the action of $\Gamma$ on $\tau$. Define $\rho_{0}(g)=\rho(g)$ and $\rho_{1}(g)=\rho\left(s_{1 / 2}(g)\right)$ for all $g \in s_{2}(\Gamma)$. Then $f_{0}$ and $f_{1}$ are equivariant with respect to $\rho_{0}$ and $\rho_{1}$ respectively.

In particular, $\tau_{0}$ and $\tau_{1}$ descend to triangulations of $M:=s_{2}(\Gamma) \backslash G$. Since this is a smooth manifold, we can construct a triangulation $(\bar{\eta}, \bar{g}: \bar{\eta} \rightarrow M \times[0,1])$ which restricts to $s_{2}(\Gamma) \backslash \tau_{i}$ on $M \times\{i\}$. This lifts to the required triangulation of $G \times[0,1]$.

Since $g_{i j}=\exp v_{i j}$ and $v_{i j}$ is in the first layer of the grading of $f_{r, n}$, we have $s_{2}\left(g_{i j}\right)=\exp 2 v_{i j}=g_{i j}^{2}$ and thus $s_{2}\left(\Lambda_{r, n}\right) \subset \Lambda_{r, n}$. The lemma thus applies to $\Lambda_{r, n}$, and we can let $\tau$ and $\eta$ be the triangulations of $F_{r, n}$ and $F_{r, n} \times[0,1]$ which are given by the lemma.

Now we can define $\phi$. For each vertex $v$ of $\tau$, let $\phi(v)$ be an element of $\Lambda_{r, n}$; we can choose these elements in a $\Lambda_{r, n}$-equivariant way. If $e$ is an edge of $\tau$ with vertices $x$ and $y$, we can choose a word $w=w\left(\phi(x)^{-1} \phi(y)\right)$ in $\Lambda_{r, n}$ which represents $\phi(x)^{-1} \phi(y)$. Then $\gamma_{w}$ is a curve connecting $e$ to $\phi(x)^{-1} \phi(y)$, and we can define $\phi(e)$ to be the translation $\phi(e)=\phi(x) \cdot \gamma_{w}$. Since $w$ depends only on $\phi(x)^{-1} \phi(y)$, this definition is also equivariant. The map $\phi$ then sends the boundary of each 2-cell $\Delta$ of $\tau$ to a curve $g \cdot \gamma_{w^{\prime}(\Delta)}$, where $w^{\prime}(\Delta)$ is a word representing the identity. By Lemma 4.4 , we can extend $\phi$ to a horizontal map on $\Delta$, and furthermore, we can do this in an equivariant way. Doing this for every cell in the 2 -skeleton of $\tau$ gives us an equivariant horizontal map on $\tau^{(2)}$, and we can extend it to an equivariant 2-horizontal map on all of $\tau$.

Next, we define $\psi$ in the same fashion. This time, because of the relationship between $\tau$ and $\eta$, many of our choices are already made for us. Let $\tau_{i}$ and $f_{i}, i=0,1$, be as in Lemma 4.5 and let $\iota_{i}: \tau_{i} \rightarrow \eta, i=0,1$, be the inclusions of the $\tau_{i}$ into $\eta$. Let

$$
\left.\psi\right|_{\iota_{0}\left(\tau_{0}\right)}=\phi \circ \iota_{0}^{-1}
$$

and

$$
\left.\psi\right|_{\iota_{1}\left(\tau_{1}\right)}=s_{2} \circ \phi \circ \iota_{1}^{-1}
$$


These definitions are $s_{2}\left(\Lambda_{r, n}\right)$-equivariant and if $v$ is a vertex of $\iota_{i}\left(\tau_{i}\right), i=0,1$, then $\phi(v) \in \Lambda_{r, n}$. We extend $\psi$ to the rest of the 1-skeleton of $\eta$ in the same way as before. For each vertex $v$ of $\eta$ which is not in $\tau_{0}$ or $\tau_{1}$, we choose an element $\psi(v) \in \Lambda_{r, n}$. For each edge $e=(x, y)$ of $\eta$ which is not in $\tau_{0}$ or $\tau_{1}$, we let

$$
\psi(e)=\psi(x) \cdot \gamma_{w\left(\psi(x)^{-1} \psi(y)\right)} .
$$

Then if $e$ is an edge of $\eta$, then $\psi(e)$ is a reparameterization of $\gamma_{w}$ for some word $w$; this is true by construction if $e$ is not in $\tau_{1}$, and if $e$ is an edge of $\tau_{1}$, then $\psi(e)$ is a curve of the form $s_{2} \circ \gamma_{w}$ for some $w=w_{1} \ldots w_{p}$. Since each of the curves making up $\gamma_{w}$ is a segment of a horizontal one-parameter subgroup, $s_{2} \circ \gamma_{w}$ is a reparameterization of $\gamma_{z}$, where $z=w_{1}^{2} \ldots w_{p}^{2}$. Thus $\psi$ sends the boundary of each 2-cell $\Delta$ of $\eta$ to a reparameterization of a curve $g \cdot \gamma_{w^{\prime}(\Delta)}$, where $w^{\prime}(\Delta)$ is a word representing the identity. Using Lemma 4.4, we can extend $\psi$ to a horizontal map on $\Delta$. Finally, we extend $\psi$ to a 2-horizontal map on all of $\eta$. All of the above extensions can be made equivariantly and Lipschitz-ly, so that $\phi$ and $\psi$ satisfy the conditions of Lemma 3.4 for $k=1$. By Theorem 5 , we conclude that $F_{r, n}$ and $\Lambda_{r, n}$ both satisfy quadratic filling inequalities when $n \geq 2$; this proves Proposition 4.1.

As a corollary, we see that the higher-dimensional Heisenberg groups, $\Lambda_{2, n}, n \geq$ 2, have quadratic Dehn functions; this result was first proved by Gromov [13] and subsequently using different methods by Ol'shanskii and Sapir [17] and Allcock [1].

Other central products of nilpotent groups can be written as quotients of the $\Lambda_{r, n}$, which lets us prove bounds on their Dehn functions as well. The following two propositions concern quotients of 2-step nilpotent groups with quadratic Dehn functions by central subgroups. They show that any such quotient has a Dehn function at most $\ell^{2} \log \ell$, and that a quotient by a commutator has quadratic Dehn function. These results complement a theorem of Wenger [22] that states that quotients of nilpotent Lie groups by central subgroups that are not generated by commutators cannot have quadratic Dehn functions. Indeed, combining Wenger's theorem and Corollary 4.7 gives a criterion for deciding whether a quotient of a 2-step group with quadratic Dehn function also has quadratic Dehn function.

In particular, the central products of the quaternionic and octonionic Heisenberg groups satisfy the hypotheses of Corollary 4.7. These groups appear as cusp groups in finite-volume rank 1 locally symmetric spaces, and Gromov [13] showed that they have quadratic Dehn functions using microflexibility. We will use Corollary 4.7 to give an alternate combinatorial proof of this fact.

Proposition 4.6. Let $G$ be a 2-step nilpotent Lie group with quadratic Dehn function and let $\mathfrak{g}$ be its Lie algebra. If $w_{1}, w_{2} \in \mathfrak{g}$, let $G^{\prime}$ be the nilpotent Lie group with Lie algebra $\mathfrak{g} /\left\langle\left[w_{1}, w_{2}\right]\right\rangle$. Then $G^{\prime}$ also has quadratic Dehn function.

Corollary 4.7. Let $G$ be a 2-step nilpotent Lie group with quadratic Dehn function and let $\mathrm{g}$ be its Lie algebra. If $W$ is generated by elements of the form $\left[w_{1}, w_{2}\right]$ with 
$w_{1}, w_{2} \in \mathfrak{g}$, let $G^{\prime}$ be the nilpotent Lie group with Lie algebra $\mathfrak{g} / W$. Then $G^{\prime}$ has quadratic Dehn function.

Proposition 4.8. Let $G$ be a nilpotent Lie group with quadratic Dehn function and let $\mathrm{g}$ be its Lie algebra. If $\mathrm{g}$ has a grading $\mathrm{g}=V_{1} \oplus V_{2}$ and if $W \subset V_{2}$, let $G^{\prime}$ be the nilpotent Lie group with Lie algebra $\mathrm{g} / W$. Then

$$
\delta_{G^{\prime}}(\ell) \preceq \ell^{2} \log \ell .
$$

Corollary 4.9. Let $G$ be a 2-step nilpotent Lie group and let $n \geq 2$. If $G^{\prime}=G^{\times} Z^{n}$, then

$$
\delta_{G^{\prime}}(\ell) \preceq \ell^{2} \log \ell .
$$

Proof. If the abelianization of $G$ has rank $d$, we can write $G=F_{d} / R$ for some $R \subset\left[F_{d}, F_{d}\right]$ and apply Proposition 4.8 to the quotient $G^{\prime}=F_{d}^{\times Z^{n}} / R$.

This corollary was first stated without proof by Ol'shanskii and Sapir [17].

The proofs of both of the propositions use the following construction, which lifts curves in $G^{\prime}$ to curves in $G$. Let $G, G^{\prime}$, and $W$ be as in Proposition 4.8, and let $p: G \rightarrow G^{\prime}$ be the quotient map. Let $g$ and $\mathrm{g}^{\prime}$ be the Lie algebras of $G$ and $G^{\prime}$ respectively, so that $\mathrm{g}=V_{1} \oplus V_{2}$ and $\mathrm{g}^{\prime}=V_{1} \oplus V_{2} / W$. We distinguish brackets in $\mathfrak{g}$ and $\mathrm{g}^{\prime}$ by writing $[\cdot, \cdot]$ or $[\cdot, \cdot]^{\prime}$ respectively.

Choose metrics on $V_{1}$ and $V_{2}$. These induce left-invariant metrics on $\mathfrak{g}$ and $\mathfrak{g}^{\prime}$. If $W^{\perp} \subset \mathrm{g}$ is the orthogonal complement of $W$, then $W^{\perp}$ is naturally isometric to $\mathrm{g}^{\prime}=\mathrm{g} / W$, and we can define $i: \mathfrak{g}^{\prime} \rightarrow \mathfrak{g}$ to be that inclusion. Define $p_{W}: V_{2} \rightarrow W$ to be the orthogonal projection to $W$.

For each Lipschitz curve $\gamma:[0, t] \rightarrow G^{\prime}$, we will define a lift $\tilde{\gamma}:[0, t] \rightarrow G$ in such a way that if $\gamma$ is $C^{1}$ and $\dot{\gamma}(t) \in \mathrm{g}^{\prime}$ is its tangent vector, we will have $\dot{\gamma}=i(\dot{\gamma})$. Let $\gamma:[0, t] \rightarrow G^{\prime}$ be a Lipschitz curve and let $\gamma_{1}:[0, t] \rightarrow V_{1}$ and $\gamma_{2}:[0, t] \rightarrow V_{2} / W$ be such that

$$
\gamma(t)=\exp \left(\gamma_{1}(t)+\gamma_{2}(t)\right)
$$

for all $t$. If $\gamma$ is $C^{1}$, then

$$
\dot{\gamma}(t)=\dot{\gamma}_{1}(t)+\dot{\gamma}_{2}(t)-\frac{1}{2}\left[\gamma_{1}(t), \dot{\gamma}_{1}(t)\right]^{\prime} .
$$

Define

$$
\tilde{\gamma}(t):=\exp \left[\gamma_{1}(t)+i\left(\gamma_{2}(t)\right)+\frac{1}{2} \int_{0}^{t} p_{W}\left(\left[\gamma_{1}(x), \dot{\gamma}_{1}(x)\right]\right) d x\right] .
$$

If $\gamma$ is $C^{1}$, we have

$$
\begin{aligned}
\dot{\tilde{\gamma}}(t) & =\dot{\gamma}_{1}(t)+i\left(\dot{\gamma}_{2}(t)\right)+\frac{1}{2}\left(p_{W}\left(\left[\gamma_{1}(t), \dot{\gamma}_{1}(t)\right]\right)-\left[\gamma_{1}(t), \dot{\gamma}_{1}(t)\right]\right) \\
& =\dot{\gamma}_{1}(t)+i\left(\dot{\gamma}_{2}(t)\right)-\frac{1}{2} i\left(\left[\gamma_{1}(t), \dot{\gamma}_{1}(t)\right]^{\prime}\right)=i(\dot{\gamma}(t)) .
\end{aligned}
$$

The following properties are easy to check: 
Lemma 4.10. If $\gamma:[0, t] \rightarrow G^{\prime}$ is a Lipschitz curve, then

- $p \circ \tilde{\gamma}=\gamma$,

- $\ell(\tilde{\gamma})=\ell(\gamma)$,

- if $\gamma:[0, t] \rightarrow G^{\prime}$ is a closed curve such that $\gamma(0)=\gamma(t)=0$, then $\tilde{\gamma}$ is a curve connecting 0 and $\exp w$ for some $w \in W$. There is a c depending only on $G$, $W$, and the chosen metrics such that $\|w\| \leq c \ell(\gamma)^{2}$.

Proof of Proposition 4.6. Since $\mathrm{g}$ is a 2-step nilpotent Lie algebra, it has a grading $\mathrm{g}=V_{1} \oplus V_{2}$. Without loss of generality, we may assume that $w_{1}, w_{2} \in V_{1}$; if not, we can replace them by their projections to $V_{1}$. Note that $w_{1}$ and $w_{2}$ generate a 2-dimensional subalgebra of $\mathfrak{g} /\left\langle\left[w_{1}, w_{2}\right]\right\rangle$.

Let $\gamma:[0,1] \rightarrow G^{\prime}$ be a closed curve such that $\gamma(0)=0$. We will construct a filling of $\gamma$. Note that $\tilde{\gamma}$ is a curve connecting 0 and $\exp \alpha\left[w_{1}, w_{2}\right]$ for some $\alpha$. If $\alpha<0$, we can reverse $\gamma$ to get a curve such that $\alpha>0$, so we may assume that $\alpha \geq 0$.

Define $q:[0,4] \rightarrow G^{\prime}$ to be the boundary of a square of area $\alpha$ in the subgroup generated by $w_{1}$ and $w_{2}$ :

$$
q(t):=\exp \begin{cases}\sqrt{\alpha} t w_{1}, & 0 \leq t \leq 1, \\ \sqrt{\alpha}\left(w_{1}+(t-1) w_{2}\right), & 1<t \leq 2, \\ \sqrt{\alpha}\left(w_{1}+w_{2}-(t-2) w_{1}\right), & 2<t \leq 3, \\ \sqrt{\alpha}\left(w_{2}-(t-3) w_{2}\right), & 3<t \leq 4\end{cases}
$$

Then $\tilde{q}:[0,4] \rightarrow G$ is a curve of length $4 \sqrt{\alpha}=O(\ell(\gamma))$ which connects 0 to $\exp \alpha\left[w_{1}, w_{2}\right]$.

If $\gamma_{1}$ and $\gamma_{2}$ are curves in $G$ with the same endpoints, then the union of $\gamma_{1}$ and $\gamma_{2}$ is a closed curve. This has a filling by a disc of area $\preceq \delta_{G}\left(\ell\left(\gamma_{1}\right)+\ell\left(\gamma_{2}\right)\right)$, and this disc can be reparameterized to be a homotopy between $\gamma_{1}$ and $\gamma_{2}$ which leaves their endpoints fixed.

Since $\tilde{\gamma}$ and $\tilde{q}$ have the same endpoints, there is a homotopy $h$ from $\tilde{\gamma}$ to $\tilde{q}$ which leaves the endpoints fixed and which has area

$$
\text { area } h \leq \delta_{G}(\ell(\tilde{q})+\ell(\tilde{\gamma}))=O\left(\ell(\gamma)^{2}\right) .
$$

This projects to a homotopy $h^{\prime}$ between $\gamma$ and $q$. Since $\left[w_{1}, w_{2}\right]=0$ in $G^{\prime}, q$ is the boundary of a square in $G^{\prime}$ of area $\alpha$ in the $w_{1} w_{2}$-plane. We can fill $\gamma$ by gluing this square to $h^{\prime}$, so

$$
\delta_{G^{\prime}}(\gamma) \leq \text { area } h^{\prime}+\alpha=O\left(\ell(\gamma)^{2}\right),
$$

as desired.

Proof of Proposition 4.8. If $\gamma_{1}:[0,1] \rightarrow G$ and $\gamma_{2}:[0,1] \rightarrow G$ are two curves in $G$ such that $\gamma_{1}(0)=\gamma_{2}(0)=0$, define $\gamma_{1} * \gamma_{2}:[0,1] \rightarrow G$ to be the curve obtained by concatenating $\gamma_{1}$ (with domain rescaled to $[0,1 / 2]$ ) and $\gamma_{1}(1) \cdot \gamma_{2}$ (with domain 
$[1 / 2,1])$; this curve connects 0 to $\gamma_{1}(1) \gamma_{2}(1)$. Define $\gamma_{1}^{* n}$ to be the concatenation of $n$ copies of $\gamma_{1}$ parameterized so that the $i$ th copy has domain $[(i-1) / n, i / n]$.

Let $\gamma:[0,1] \rightarrow G^{\prime}$ be a closed curve such that $\gamma(0)=0$. Without loss of generality, we may assume $\ell(\gamma) \geq 1$. Then $\tilde{\gamma}(1)$ is an element of $\exp W$; let $w=$ $\log \tilde{\gamma}(1)$. For any $i \geq 0$, let $\tilde{\gamma}_{i}:=\left(s_{2-i} \circ \tilde{\gamma}\right)^{* 4^{i}}$; this too is a curve connecting 0 and $\exp w$, and we will construct a homotopy from $\tilde{\gamma}$ to $\gamma_{k}$ which passes through $\gamma_{1}, \ldots, \gamma_{k-1}$.

Since $G$ has a quadratic Dehn function, there is a homotopy $h$ between $\gamma_{0}$ and $\gamma_{1}$ which fixes the endpoints of the curves. Scalings of this homotopy can be combined to get homotopies from $\gamma_{i}$ to $\gamma_{i+1}$. Indeed, $s_{2^{-i}} \circ h$ is a reparameterization of a homotopy from

to

$$
s_{2-i} \circ \gamma_{0}=\left.\gamma_{i}\right|_{\left[0,4^{-i}\right]}
$$

$$
s_{2^{-i}} \circ \gamma_{1}=\left.\gamma_{i+1}\right|_{\left[0,4^{-i}\right]},
$$

and we can build a homotopy $h_{i}$ from $\gamma_{i}$ to $\gamma_{i+1}$ out of $4^{i}$ copies of $s_{2-i} \circ h$. This homotopy has area at most $4^{i} 4^{-i}$ area $h=$ area $h$.

Let $i_{0} \geq 0$ be an integer such that $2^{i_{0}} \leq \ell(\gamma)<2^{i_{0}+1}$ and consider the homotopy $\bar{h}$ from $\gamma_{0}$ to $\gamma_{i_{0}+1}$ obtained by concatenating $h_{0}, \ldots, h_{i_{0}-1}$. Let $p: G \rightarrow G^{\prime}$ be the quotient map and consider $p \circ \bar{h}$. This is a homotopy from $\gamma$ to $\left(s_{2}-i_{0} \circ \gamma\right)^{* 4^{i} 0}$ with area $\leq i_{0}$ area $h=O\left((\ell(\gamma))^{2} \log \ell(\gamma)\right)$. The curve $s_{2-i_{0}} \circ \gamma$ is a closed curve with length $\leq 1$, so it has filling area $\leq \delta_{G^{\prime}}(1)$ and the concatenation $\left(s_{2^{-i_{0}}} \circ \gamma\right)^{* 4^{i_{0}}}$ has filling area $\leq 4^{i_{0}} \delta_{G^{\prime}}(1)=O\left((\ell(\gamma))^{2}\right)$. Combining $p \circ \bar{h}$ with a filling of $\left(s_{2}-i_{0} \circ \gamma\right)^{* 4^{i} 0}$ results in a filling of $\gamma$ with area $O\left((\ell(\gamma))^{2} \log \ell(\gamma)\right)$.

Two families of groups that satisfy the conditions of Corollary 4.7 are the higherdimensional quaternionic and octonionic Heisenberg groups; these groups appear as cusp groups in finite-volume quaternion-hyperbolic and Cayley-hyperbolic manifolds. These groups can be defined as follows: let $\mathbb{K}=\mathbb{C}, \mathbb{H}, \mathbb{O}$ be the complex numbers, quaternions, or octonions and let $\mathbb{K}^{*}$ be the elements of $\mathbb{K}$ with real part 0 (the pure imaginary elements of $\mathbb{K}$ ). We can then define a graded nilpotent Lie algebra $\mathfrak{h}_{\mathbb{K}}=\mathbb{K} \oplus \mathbb{K}^{*}$. Its bracket is given by a bilinear form $\omega: \mathbb{K} \times \mathbb{K} \rightarrow \mathbb{K}^{*}$, which we define as $\omega(v, w)=\mathfrak{s}(v \bar{w})$, where $\Im(x)$ is the pure imaginary part of $x$. Then $\mathfrak{h}_{\mathbb{K}}$ is a 2-step graded nilpotent Lie algebra, and we define $H_{\mathbb{K}}$ to be the corresponding nilpotent Lie group. We call $H_{\mathbb{C}}$ the Heisenberg group, and call $H_{\mathbb{H}}$ and $H_{\mathbb{O}}$ the quaternionic and octonionic Heisenberg groups. Gromov [13] showed that $H_{\mathbb{C}}$ has a cubic Dehn function; Pittet [18] extended this result to $H_{\mathbb{H}}$, but the Dehn function of $H_{\mathbb{O}}$ is unknown (it was claimed to be cubic in [18], but the calculation contained a sign error, corrected in [15]).

Central products of the (quaternionic or octonionic) Heisenberg group are called higher-dimensional (quaternionic or octonionic) Heisenberg groups. The corresponding Lie algebras have presentations based on the multiplication table of $\mathbb{K}$. For 
instance, $\mathbb{H}$ is generated as a vector space by unit quaternions $1, i, j, k$, with multiplication table

\begin{tabular}{r|rrrr} 
& 1 & $i$ & $j$ & $k$ \\
\hline 1 & 1 & $i$ & $j$ & $k$ \\
$i$ & $i$ & -1 & $k$ & $-j$ \\
$j$ & $j$ & $-k$ & -1 & $i$ \\
$k$ & $k$ & $j$ & $-i$ & -1.
\end{tabular}

If we let $\digamma_{4}$ be the free 2-step Lie algebra of rank 4 and denote its generators by $1, i, j$, and $k$, we can write $\mathfrak{h}_{\mathbb{H}}$ as a quotient of $\mathfrak{f}_{4}$ by identifying brackets of the generators in pairs:

$$
\mathfrak{h}_{\mathbb{H}}=\mathfrak{f}_{4} /\langle[1, i]-[j, k],[1, j]-[k, i],[1, k]-[i, j]\rangle .
$$

If we denote the generators of $\mathfrak{f}_{4, n}$ by $1_{1}, i_{1}, j_{1}, k_{1}, \ldots, 1_{n}, i_{n}, j_{n}, k_{n}$, we can write $\mathfrak{h}_{\mathbb{H}}^{\times Z n}$ as a quotient of $\mathfrak{f}_{4, n}$ :

$$
\begin{aligned}
\mathfrak{h}_{\mathbb{H}}^{\times Z n} & =\mathfrak{f}_{4, n} /\left\langle\left[1_{1}, i_{1}\right]-\left[j_{2}, k_{2}\right],\left[1_{1}, j_{1}\right]-\left[k_{2}, i_{2}\right],\left[1_{1}, k_{1}\right]-\left[i_{2}, j_{2}\right]\right\rangle \\
& =\mathfrak{f}_{4, n} /\left\langle\left[1_{1}+j_{2}, i_{1}-k_{2}\right],\left[1_{1}+k_{2}, j_{1}-i_{2}\right],\left[1_{1}+i_{2}, k_{1}-j_{2}\right]\right\rangle .
\end{aligned}
$$

Since this is a quotient by commutators, Corollary 4.7 applies, and $H_{\mathbb{H}}^{\times Z^{n}}$ has a quadratic Dehn function. The group $H_{\mathbb{O}}^{\times Z^{n}}$ has a similar presentation and thus also has a quadratic Dehn function.

4.2. Jet groups. In [14], 4.1.D, 4.4.A-B], Gromov used infinitesimal invertibility and the $h$-principle to prove a Lipschitz Extension Theorem for many spaces, including the $k$-jet bundle. This theorem can be used to construct maps and triangulations satisfying Theorem 3 for many groups. In the case of the $k$-jet bundle, these maps and triangulations can be constructed fairly explicitly (see also [19]), and in this section, we provide an elementary construction of such maps and triangulations for jet groups, a family of groups based on jet bundles which includes the higher-dimensional Heisenberg groups.

This family of groups has also appeared as a family of non-rigid Carnot groups [20] and a family of quadratically presented Lie algebras [7]. Warhurst defined the group by putting a group structure on the $m$-jet bundle $J^{m}\left(\mathbb{R}^{k}\right)$; we will give a version of this construction.

The $m$-jet bundle is a generalization of the cotangent bundle and is often used to describe differential relations. A smooth map from $M$ to $\mathbb{R}$ has a gradient which can be considered as a map from $M$ to the cotangent bundle, $T M^{*}$. Likewise, its $m$-th order derivative can be considered as a map to the $i$ th symmetric power of $T M^{*}$. The $m$-jet bundle is a sum of these symmetric powers. We will mainly consider the $m$-jet bundle of $\mathbb{R}^{k}$, which we denote by $J^{m}\left(\mathbb{R}^{k}\right)$. This is a vector bundle with fiber

$$
W=\bigoplus_{i=0}^{m} W_{i},
$$


where $W_{i}:=S^{i} \mathbb{R}^{k *}$ is the $i$ th symmetric power of $\mathbb{R}^{k *}$ and $W_{0}:=S^{0} \mathbb{R}^{k *}=\mathbb{R}$. A $C^{n}$ map $f: \mathbb{R}^{k} \rightarrow \mathbb{R}$ corresponds to a $C^{n-m}$ section, called a prolongation, $j^{m}(f): \mathbb{R}^{k} \rightarrow J^{m}\left(\mathbb{R}^{k}\right)$, given by taking derivatives: the projection to $W_{0}$ corresponds to the original map, the projection to $W_{1}=\mathbb{R}^{k *}$ is the gradient of $f$, and so on. We will often write $j_{p}^{m}(f)$ in place of $j^{m}(f)(p)$. In the Carnot structure that we will construct on $J^{m}\left(\mathbb{R}^{k}\right)$, prolongations of smooth functions will be horizontal.

Using a basis of $\mathbb{R}^{k}$, we can construct a basis of $W$. Let $\left\{e_{1}, \ldots, e_{k}\right\}$ be the standard basis of $\mathbb{R}^{k}$, and let $\left\{e_{1}^{*}, \ldots, e_{k}^{*}\right\}$ be the corresponding basis of $\mathbb{R}^{k *}$. If we let

$$
y_{\left(a_{1}, \ldots, a_{k}\right)}=\prod_{i=1}^{k}\left(e_{i}^{*}\right)^{a_{i}},
$$

then

$$
\left\{y_{\left(a_{1}, \ldots, a_{k}\right)} \mid \sum a_{i}=n\right\}
$$

is a basis of $W_{n}$ and

$$
\left\{y_{\left(a_{1}, \ldots, a_{k}\right)} \mid \sum a_{i} \leq m\right\}
$$

is a basis of $W$. Using this basis, we can write $J^{m}\left(\mathbb{R}^{k}\right)$ as a product $J^{m}\left(\mathbb{R}^{k}\right)=$ $\mathbb{R}^{k} \times W$.

We can now define the group structure. First, note that for every $p \in J^{m}\left(\mathbb{R}^{k}\right)$, there is a unique polynomial in $k$ variables of degree at most $m$ whose prolongation passes through $p$. We call this polynomial $P(p)$, and we use it to construct an action of $\mathbb{R}^{k}$ on $W$. Define $D_{x}^{m}(f) \in W$ to be the first $m$ derivatives of $f$ at $x$, so that

$$
j_{x}^{m}(f)=\left(x, D_{x}^{m}(f)\right) .
$$

If $x \in \mathbb{R}^{k}$ and $w \in W$, we let $S_{x}: W \rightarrow W$ be the map $S_{x}(w)=D_{x}^{m}(P((0, w)))$. This is an action of $\mathbb{R}^{k}$ on $W$, and we define

$$
\left(p_{1}, p_{2}\right)\left(q_{1}, q_{2}\right)=\left(p_{1}+q_{1}, S_{q_{1}}\left(p_{2}\right)+q_{2}\right) .
$$

This makes $J^{m}\left(\mathbb{R}^{k}\right)$ a semidirect product of $\mathbb{R}^{k}$ and $W$. One can check that $\mathbb{R}^{k} \times\{0\}$ is a subgroup and that the translate $p \cdot\left(\mathbb{R}^{k} \times\{0\}\right)$ is the graph of $j^{m}(P(p))$.

To describe the Lie algebra $\dot{j}_{m, k}$ of $J^{m}\left(\mathbb{R}^{k}\right)$, consider the basis

$$
\left\{y_{\left(a_{1}, \ldots, a_{k}\right)} \mid \sum a_{i} \leq m\right\} \cup\left\{e_{1}, \ldots, e_{k}\right\}
$$

of $j_{m, k}$. Calculating brackets, we find that

$$
\left[e_{i}, y_{\left(a_{1}, \ldots, a_{k}\right)}\right]=y_{\left(a_{1}, \ldots, a_{i}-1, \ldots, a_{k}\right)} \quad \text { if } a_{i}>0 \text {. }
$$

and all other brackets are zero. This Lie algebra is isomorphic to the Lie algebra corresponding to the model $\mathcal{M}_{m, k}$ defined by Chen [7]. We can give $\dot{j}_{m, k}$ the grading

$$
\dot{j}_{m, k}=\left(\mathbb{R}^{k} \oplus W_{m}\right) \oplus W_{m-1} \oplus \cdots \oplus W_{0} .
$$


Moreover, since the structure constants of $j_{m, k}$ with respect to the basis $\left\{x_{i}, y_{\left(a_{1}, \ldots, a_{k}\right)}\right\}$ are rational, $\left\{\exp x_{1}, \ldots, \exp x_{k}\right\} \cup\left\{\exp y_{\left(a_{1}, \ldots, a_{k}\right)} \mid \sum a_{i}=m\right\}$ generate a lattice in $J^{m}\left(\mathbb{R}^{k}\right)$. Call this lattice $\Gamma_{m, k}$. Since the generators are in $\exp \left(\mathbb{R}^{k} \oplus W_{m}\right)$, we have $s_{2}\left(\Gamma_{m, k}\right) \subset \Gamma_{m, k}$.

Note that the groups $J^{1}\left(\mathbb{R}^{k}\right)$ are the $(2 k+1)$-dimensional Heisenberg groups and $J^{2}\left(\mathbb{R}^{2}\right)$ is the class 3 example given in [25]; one isomorphism between them takes

$$
a, b, c, d, e, f, g, h
$$

to

$$
y_{(2,0)}, x_{1}, y_{(1,1)}, x_{2}, y_{(0,2)},-y_{(1,0)}, y_{(0,1)},-y_{(0,0)},
$$

respectively.

Warhurst showed that the left-invariant plane field corresponding to $\mathbb{R}^{k} \oplus W_{m}$ agrees with the standard contact structure on $J^{m}\left(\mathbb{R}^{k}\right)$ [20]. This gives a way to construct horizontal submanifolds: if $U$ is an open subset of $\mathbb{R}^{k}$ and $f: U \rightarrow \mathbb{R}$ is smooth, we define $M_{f}$ to be the image of $j^{m}(f): U \rightarrow J^{m}\left(\mathbb{R}^{k}\right)$. Then $M_{f}$ is a horizontal submanifold:

Lemma 4.11. If $U$ is an open subset of $\mathbb{R}^{k}$ and $f: U \rightarrow \mathbb{R}$ is smooth, then $M_{f}$ is a smooth horizontal submanifold of $J^{m}\left(\mathbb{R}^{k}\right)$.

Proof. $M_{f}$ is smooth by the definition of $j^{m}(f)$, so it just remains to show that its tangent plane lies in a translate of $V_{1}=\mathbb{R}^{k} \oplus W_{m}$.

First, note that any translate of a prolongation is still a prolongation. Specifically, if $p=\left(p_{1}, p_{2}\right) \in J^{m}\left(\mathbb{R}^{k}\right)$, then $p \cdot M_{f}=M_{g}$ for $g: U^{\prime} \rightarrow \mathbb{R}$ defined by

$$
g(x)=f\left(x-p_{1}\right)+P(p)(x),
$$

where $U^{\prime}=U+p_{1}=\left\{u+p_{1} \mid u \in U\right\}$ and $P(p)(x)$ is the polynomial used above to define the group structure on $J^{m}\left(\mathbb{R}^{k}\right)$.

Let $x \in U$. If we take $p=\left[j_{x}^{m}(f)\right]^{-1}$ in the previous construction, then $g$ is a smooth function which vanishes to $m$ th order at 0 . The tangent plane to $M_{f}$ at $j_{x}^{m}(f)$ is then a translate of the tangent plane to $M_{g}$ at 0 . Since the first $m$ derivatives of $g$ disappear at 0 , the tangent plane to $M_{g}$ at 0 is contained in $\mathbb{R}^{k} \oplus W_{m}$. Indeed, if we consider the derivative of the $m$ th derivative of $g$ as a map from $\mathbb{R}^{k} \rightarrow W_{m}$, then the tangent plane to $M_{g}$ is the graph of this map.

Now we define a class of horizontal manifolds coming from the construction of Lemma 4.11.

Definition 1. If $U \subset \mathbb{R}^{k}$ is an open subset and $f: U \rightarrow \mathbb{R}$ is a smooth map, we say that any submanifold $Y$ of $M_{f}$ is holonomic.

If $X$ is a complex, a Lipschitz map $f: X \rightarrow J^{m}\left(\mathbb{R}^{k}\right)$ is holonomic if and only if its image lies in $M_{f}$ for some smooth $f: U \rightarrow \mathbb{R}$. 
Lemma 4.11 then implies that holonomic submanifolds and maps are horizontal.

A cycle in a holonomic submanifold is equipped with a smooth function, and we can use this to construct a holonomic filling. That is, if $f: U \rightarrow \mathbb{R}$ is a smooth function defined on an open set and if $\alpha$ is a singular Lipschitz cycle in $M_{f}$, then we can construct a holonomic filling of $\alpha$. The support of $\alpha$ is compact, so there is a smooth function $\bar{f}: \mathbb{R}^{k} \rightarrow \mathbb{R}$ which agrees with $f$ on a neighborhood of the support of $\alpha$. In particular, we can write $\alpha$ as $J^{m}(\bar{f})_{\sharp}\left(\alpha_{0}\right)$ for some cycle $\alpha_{0}$ in $\mathbb{R}^{k}$. If $\beta_{0}$ is a chain in $\mathbb{R}^{k}$ which fills $\alpha_{0}$, then $J^{m}(\bar{f})_{\sharp}\left(\beta_{0}\right)$ is a chain in $J^{m}\left(\mathbb{R}^{k}\right)$ which fills $\alpha$.

In general, a horizontal map is not necessarily even locally holonomic, and it can be difficult to fill an arbitrary horizontal map with a horizontal filling. For our purposes, it suffices to fill locally holonomic maps. To work with such maps, we will define augmented maps which are horizontal maps locally equipped with smooth functions.

Definition 2. Let $X$ be a simplicial complex. Let $p_{\mathbb{R}^{k}}: J^{m}\left(\mathbb{R}^{k}\right) \rightarrow \mathbb{R}^{k}$ be the bundle projection. An augmented map from $X$ to $J^{m}\left(\mathbb{R}^{k}\right)$ is a tuple

$$
\left(\alpha: X \rightarrow J^{m}\left(\mathbb{R}^{k}\right),\left\{f_{\Delta}: V_{\Delta} \rightarrow \mathbb{R}\right\}_{\Delta \subset X}\right)
$$

which satisfies two conditions. First, we require that the map $\alpha$ is holonomic on each cell. That is, the image of a cell $\Delta$ is contained in $M_{f_{\Delta}}$. Second, we require that if $\Delta_{1}$ is a face of $\Delta_{2}$, then $M_{f_{\Delta_{1}}} \subset M_{f_{\Delta_{2}}}$. Note that this imposes compatibility conditions on any pair of faces that intersect, because if $\Delta$ and $\Delta^{\prime}$ intersect, then $M_{f_{\Delta \cap \Delta^{\prime}}} \subset M_{f_{\Delta}}$ and $M_{f_{\Delta \cap \Delta^{\prime}}} \subset M_{f_{\Delta^{\prime}}}$, so $f_{\Delta}$ and $f_{\Delta^{\prime}}$ agree on a neighborhood of $\Delta \cap \Delta^{\prime}$.

If $X$ is a subcomplex of $Y$ and if $\left(\alpha,\left\{f_{\Delta}\right\}_{\Delta \subset X}\right)$ is an augmented map on $X$, we say that $\left(\beta,\left\{g_{\Delta}\right\}_{\Delta \subset Y}\right)$ extends $\left(\alpha,\left\{f_{\Delta}\right\}\right)$ if and only if $\beta$ extends $\alpha$ in the ordinary sense and $M_{g_{\Delta}} \subset M_{f_{\Delta}}$ for all simplices $\Delta \subset X$. If $\kappa: X \rightarrow \mathbb{R}^{k}$, then we say that $\left(\alpha,\left\{f_{\Delta}\right\}\right)$ covers $\kappa$ if $p_{\mathbb{R}^{k}} \circ \alpha=\kappa$.

Lemma 4.12. If $\kappa: \Delta \rightarrow \mathbb{R}^{k}$ is a Lipschitz embedding of a simplex and

$$
\left(\alpha,\left\{f_{\delta}: V_{\delta} \rightarrow \mathbb{R}\right\}_{\delta \subset \partial \Delta}\right)
$$

is an augmented map on $\partial \Delta$ which covers $\left.\kappa\right|_{\partial \Delta}$, then there is an augmented map $\left(\beta,\left\{g_{\delta}: W_{\delta} \rightarrow \mathbb{R}\right\}_{\delta \subset \Delta}\right)$ which extends $\left(\alpha,\left\{f_{\delta}\right\}\right)$ and covers $\kappa$.

Proof. It suffices to find a smooth function $g: \mathbb{R}^{k} \rightarrow \mathbb{R}$ such that $g$ agrees with $f_{\delta}$ on a neighborhood of $\kappa(\delta)$ for each face $\delta$. If we have such a $g$, we can construct the required extension by letting $g_{\Delta}=g, \beta=J^{m}(g) \circ \kappa$, and $W_{\Delta}=\mathbb{R}^{k}$. To find $g$, we use a partition of unity.

If $\delta$ is a face of $\Delta$, let

$$
U_{\delta}:=V_{\delta}-\bigcup_{\delta^{\prime} \not \supset \delta} \kappa\left(\delta^{\prime}\right) \quad \text { for all } \delta \subsetneq \Delta,
$$


and let $U_{\Delta}=\mathbb{R}^{k}-\kappa(\partial \Delta)$. For each $\delta$, we have $\kappa($ int $\delta) \subset U_{\delta}$, so the $U_{\delta}$ 's form an open cover of $\mathbb{R}^{k}$. Let $\left\{\rho_{\delta}: \mathbb{R}^{k} \rightarrow \mathbb{R}\right\} \delta \subset \Delta$ be a smooth partition of unity subordinate to $\left\{U_{\delta}\right\}$.

Let $f_{\Delta}: \mathbb{R}^{k} \rightarrow \mathbb{R}$ be a smooth function and let $g: \mathbb{R}^{k} \rightarrow \mathbb{R}$ be $g=\sum_{\delta} \rho_{\delta} f_{\delta}$. We claim that if $\delta$ is a simplex of $\Delta$, then $g$ agrees with $f_{\delta}$ on some neighborhood of $\kappa(\delta)$. Let $x \in \kappa(\delta)$. If $x \in \operatorname{supp} \rho_{\delta^{\prime}}$, then $x \in U_{\delta^{\prime}}$. Furthermore, $U_{\delta^{\prime}} \cap \kappa(\delta)=\emptyset$ when $\delta^{\prime} \not \subset \delta$, so if $x \in \operatorname{supp} \rho_{\delta^{\prime}}$, then $\delta^{\prime} \subset \delta$. There is thus a neighborhood $U$ of $x$ such that

$$
\left.g\right|_{U}=\sum_{\delta^{\prime} \subset \delta} \rho_{\delta^{\prime}} f_{\delta^{\prime}}
$$

All of the $f_{\delta^{\prime}}$ agree with $f_{\delta}$ on a neighborhood of $x$, so $g$ agrees with $f_{\delta}$ on a neighborhood of $x$. Since this is true for any $x \in \kappa(\delta)$, we conclude that $f_{\delta}$ and $g$ agree on some neighborhood of $\kappa(\delta)$. We can thus construct the required extension by letting $g_{\Delta}=g$ and $\beta=J^{m}(g) \circ \kappa$.

This lets us construct maps satisfying the conditions of Theorem 3 by induction on dimension. We first define an action of $J^{m}\left(\mathbb{R}^{k}\right)$ on augmented maps. Let $p=$ $\left(p_{1}, p_{2}\right) \in J^{m}\left(\mathbb{R}^{k}\right)$. Define

$$
p \cdot\left(\alpha,\left\{f_{\Delta}\right\}_{\Delta \subset X}\right)=\left(p \cdot \alpha,\left\{p \cdot f_{\Delta}\right\}_{\Delta \subset X}\right)
$$

where

$$
\begin{aligned}
(p \cdot \alpha)(x) & =p \alpha(x), \\
\left(p \cdot f_{\Delta}\right)(x) & =f_{\Delta}\left(x-p_{1}\right)+P(p)(x) .
\end{aligned}
$$

It is easily checked that this is a group action on the space of augmented maps.

Lemma 4.13. There are $\tau, \eta, \phi$ and $\psi$ satisfying the conditions of Theorem 3 for $G=J^{m}\left(\mathbb{R}^{k+1}\right)$ and $k=k$.

Proof. Let $\Gamma=\Gamma_{m, k+1}$ and recall that $s_{2}(\Gamma) \subset \Gamma$. Let $p: J^{m}\left(\mathbb{R}^{k+1}\right) \rightarrow \mathbb{R}^{k+1}$ be the bundle projection and let $(\tau, f: \tau \rightarrow G)$ be a $\Gamma$-equivariant triangulation of $G$ and let $(\eta, g: \eta \rightarrow G \times[0,1])$ be an $s_{2}(\Gamma)$-equivariant triangulation of $G \times[0,1]$, as in Lemma 4.5. We will construct horizontal maps $\phi: \tau \rightarrow G$ and $\psi: \eta \rightarrow G$ by constructing augmented maps on the $k+1$-skeletons of $\tau$ and $\eta$.

We can construct an action of $\Gamma$ on $\mathbb{R}^{k+1}$ by letting the action of $\gamma$ send $x \mapsto$ $x+p(\gamma)$. After possibly subdividing $\tau$, we can construct a $\Gamma$-equivariant map $\kappa: \tau \rightarrow \mathbb{R}^{k+1}$ so that the vertices of any simplex of $\tau$ lie in general position and each simplex is mapped linearly to $\mathbb{R}^{k+1}$; this is an embedding on each simplex in $\tau^{(k+1)}$.

We can now use Lemma 4.12 to build a $\Gamma$-equivariant augmented map on the 0 -skeleton, then the 1 -skeleton, and so on up to the $(k+1)$-skeleton. This constructs a horizontal $\Gamma$-equivariant map on $\tau^{(k+1)}$ which can then be extended to all of $\tau$.

We construct $\psi$ by a similar process. The main difference is the starting point; if $\tau_{0}$ and $\tau_{1}$ are as in Lemma 4.5 and if $\iota_{i}: \tau_{i} \rightarrow \eta, i=0,1$, are the inclusions of the 
$\tau_{i}$ into $\eta$, we define $\psi_{0}: \tau_{0} \cup \tau_{1} \rightarrow G$ by

$$
\left.\psi_{0}\right|_{\iota_{0}\left(\tau_{0}\right)}=\phi \circ \iota_{0}^{-1}
$$

and

$$
\left.\psi_{0}\right|_{\iota_{1}\left(\tau_{1}\right)}=s_{2} \circ \phi \circ \iota_{1}^{-1}
$$

and extend this to an $s_{2}(\Gamma)$-equivariant $(k+1)$-horizontal map on $\eta$.

We thus have

Theorem 6. $J^{m}\left(\mathbb{R}^{k}\right)$ satisfies the filling inequalities

$$
\mathrm{FV}^{n}(V) \prec V^{\frac{n}{n-1}} \quad \text { for } 2 \leq n \leq k .
$$

In the case of the Heisenberg group $H_{2 k+1}=J^{1}\left(\mathbb{R}^{k}\right)$, we have

Corollary 4.14. $H_{2 k+1}$ satisfies the filling inequalities

$$
\mathrm{FV}^{n}(V) \prec V^{\frac{n}{n-1}} \quad \text { for } 2 \leq n \leq k .
$$

\section{More upper bounds}

When $G$ has no horizontal $(k+1)$-manifolds, the constructions of Section 3 still provide fillings of cycles, but these fillings satisfy weaker bounds. In some cases, however (especially when there are many horizontal $k$-manifolds), these weaker bounds may still be sharp. One notable application is a sharp upper bound for the filling volume of the higher-dimensional Heisenberg groups in the middle dimension.

Lemma 5.1. Let $k>0$. Let $\tau, \eta, \phi: \tau \rightarrow G$, and $\psi: \eta \rightarrow G$ be as in Lemma 3.4, except with no requirement that $\phi$ and $\psi$ be horizontal.

Let $f: \mathbb{R}^{+} \rightarrow \mathbb{R}^{+}$be a function such that for any $(k+1)$-simplex $\Delta$ of $\eta$, we have mass $s_{t}(\psi(\Delta)) \leq f(t)$. If, as in Lemma 3.4, we define

$$
R_{i}(\alpha):=s_{2^{i}}\left(R_{\psi(\eta)}\left(s_{2^{-i}}(\alpha)\right)\right)
$$

there is a $c$ such that for all $i \geq 0$ and for all $k$-cycles $\alpha$ we have

$$
\partial R_{i}(\alpha)=P_{i+1}(\alpha)-P_{i}(\alpha)
$$

and

$$
\operatorname{mass} R_{i}(\alpha) \leq 2^{-k i} c f\left(2^{i}\right) \operatorname{mass} \alpha
$$


Proof. The fact that

$$
\partial R_{i}(\alpha)=P_{i+1}(\alpha)-P_{i}(\alpha)
$$

follows from the proof of Lemma 3.4.

If $\beta$ is a simplicial chain in a simplicial complex $\sigma$, it can be written as $\beta=$ $\sum_{\Delta \in \sigma} b_{\Delta} \Delta$, where $\Delta$ ranges over the simplices of $\sigma$. Define

$$
\|\beta\|_{1}=\sum_{\Delta \in \sigma}\left|b_{\Delta}\right|
$$

Recall that if $\gamma$ is a Lipschitz $k$-cycle, then we defined

$$
R_{\psi(\eta)}(\gamma)=\psi_{\sharp}\left(P_{\eta}(X(\gamma))\right) .
$$

By Theorem 2, there is a $c$ depending only on $\eta$ such that $\left\|P_{\eta}(X(\gamma))\right\|_{1} \leq c$ mass $\gamma$. Therefore, we have

$$
\left\|P_{\eta}\left(X\left(s_{2-i}(\alpha)\right)\right)\right\|_{1} \leq c 2^{-k i} \operatorname{mass} \alpha,
$$

so $R_{i}(\alpha)$ is the sum of at most ( $c 2^{-k i}$ mass $\left.\alpha\right)$ singular simplices of the form $\left(s_{2^{i}}\right.$ 。 $\psi)_{\sharp}(\Delta)$, where $\Delta$ is a simplex of $\eta$. Each of these has mass $\leq f\left(2^{i}\right)$, so

$$
\operatorname{mass} R_{i}(\alpha) \leq 2^{-k i} c f\left(2^{i}\right) \operatorname{mass} \alpha .
$$

The same construction used to prove Theorem 3 can be used to prove:

Theorem 7. Let $k, \tau, \phi, \eta$, and $\psi$ satisfy the hypotheses of Lemma 5.1. Then

$$
\mathrm{FV}_{G}^{k+1}(V) \preceq \sum_{i=1}^{\left(\log _{2} V\right) / k} f\left(2^{i}\right) \frac{V}{2^{k i}} .
$$

So if $f(t) \sim t^{p}$, where $p>k$, we have

$$
\mathrm{FV}_{G}^{k+1}(V) \preceq V^{\frac{p}{k}} .
$$

To apply this to the jet groups, we use the maps constructed in Lemma 4.13. In that lemma, we constructed triangulations $\tau$ and $\eta$ and equivariant $k$-horizontal maps $\phi: \tau \rightarrow J^{m}\left(\mathbb{R}^{k}\right)$ and $\psi: \eta \rightarrow J^{m}\left(\mathbb{R}^{k}\right)$; these maps are horizontal when restricted to $\tau^{(k)}$ and $\eta^{(k)}$, and we will define equivariant maps $\phi^{\prime}$ and $\psi^{\prime}$ which extend $\left.\phi\right|_{\tau^{(k)}}$ and $\left.\psi\right|_{\eta^{(k)}}$.

Let $\Delta_{1}, \ldots, \Delta_{d}$ be a set of $(k+1)$-simplices which form a fundamental domain for $\tau^{(k+1)}$. Then $\phi^{\prime}$ is already defined on $\partial \Delta_{i}$ for each $i$ and is horizontal. We can identify $\Delta_{i}$ with the cone $C \partial \Delta_{i}:=\left(\partial \Delta_{i} \times[0,1]\right) /\left(\Delta_{i} \times\{0\}\right)$ over $\partial \Delta_{i}$ and define $h_{i}: C \Delta_{i} \rightarrow G$ as the map which sends $(x, t) \in \partial \Delta_{i} \times[0,1]$ to $s_{t}\left(\phi^{\prime}(x)\right)$. This map is not horizontal, but it scales relatively slowly; at almost every point in the image, 
the tangent plane to $h_{i}$ is the sum of a $k$-dimensional horizontal subspace and another vector. Since $J^{m}\left(\mathbb{R}^{k}\right)$ has nilpotency class $m+1$, any vector $v$ in $J^{m}\left(\mathbb{R}^{k}\right)$ satisfies $\left\|s_{t}(v)\right\| \leq t^{m+1}\|v\|$, and so we have

$$
\operatorname{mass}\left(s_{t} \circ h_{i}\right)_{\sharp}\left(\Delta_{i}\right) \leq t^{k+m+1} \operatorname{mass} h_{i \sharp}\left(\Delta_{i}\right) .
$$

Define $\left.\phi^{\prime}\right|_{\Delta_{i}}=h_{i}$ for each $i$, and extend this definition to the entire $(k+1)$ skeleton by equivariance. Finally, extend $\phi^{\prime}$ to an equivariant map on all of $\tau$. If $c=\max _{i} \operatorname{vol} h_{i}$, then this map has the property that for any $(k+1)$-simplex $\Delta$ of $\tau$, we have $\operatorname{mass}\left(s_{t} \circ \phi^{\prime}\right)_{\sharp}(\Delta) \leq c t^{k+m+1}$.

Similar techniques let us extend $\left.\psi\right|_{\eta^{(k)}}$ to a map $\psi^{\prime}: \eta \rightarrow G$ such that for any $(k+1)$-simplex $\Delta$ of $\eta$, we have mass $\left(s_{t} \circ \psi^{\prime}\right)_{\sharp}(\Delta) \leq c^{\prime} t^{k+m+1}$. We thus have

Theorem 8. $J^{m}\left(\mathbb{R}^{k}\right)$ satisfies the filling inequality

$$
\mathrm{FV}^{k+1}(V) \prec V^{\frac{k+m+1}{k}} .
$$

Corollary 5.2. $H_{2 k+1}$ satisfies the filling inequality

$$
\mathrm{FV}^{k+1}(V) \prec V^{\frac{k+2}{k}} \text {. }
$$

\section{Lower bounds}

Gromov [13], Baumslag, Miller, and Short[3], Pittet [18], and Burillo [6] have all used cohomological methods to prove lower bounds on filling functions in nilpotent groups. The idea is related to the use of calibrations in geometric measure theory: if $\omega$ is a closed $k$-cochain in a group $G$, then the value of $\omega$ on a $k$-chain $\alpha$ is determined by its boundary $\partial \alpha$. We denote this value by $\omega(\alpha)$. If $\omega$ satisfies a bound $\omega(\beta) \leq c$ mass $\beta$ for all $k$-chains $\beta$ (for instance, if $\omega$ is a left-invariant de Rham or simplicial cochain), it follows that $\mathrm{FV}^{k}(\partial \alpha) \geq \omega(\alpha) / c$.

So one can find a lower bound on $\mathrm{FV}^{k}$ by finding a $k$-cocycle $\omega$ and a $k$-chain $\alpha$ such that $\partial \alpha$ is small and $\omega(\alpha)$ is large. Gromov gives one way of finding such chains in the Heisenberg group, and we will sketch his argument below. Let $H_{3}=\mathbb{R}^{3}$ with the multiplication

$$
(x, y, z) \cdot\left(x^{\prime}, y^{\prime}, z^{\prime}\right)=\left(x+x^{\prime}, y+y^{\prime}, z+z^{\prime}+x y^{\prime}\right) .
$$

Then the 2-form $\omega=d y \wedge d z$ is closed and left-invariant, and if $S$ is an open set in the $y z$-plane, then there is a $c \neq 0$ such that $\int_{S} \omega=c$ area $S$. In particular, if $S(y, z)$ is the rectangle $\{0\} \times[0, y] \times[0, z]$, then $\int_{S(y, z)} z=c y z$, so we can use these rectangles to produce 2-chains such that $\omega(\alpha)$ is large. It remains to make $\partial \alpha$ small. Consider the closed curve $\gamma$ that connects $(0,0,0),(0, r, 0),\left(0, r, r^{2}\right)$, and $\left(0,0, r^{2}\right)$ by geodesics. Because the $z$-axis of $H$ is quadratically distorted, this curve 
has length $\sim r$, but it is close enough to the boundary of $S\left(r, r^{2}\right)$ that one can show that any 2-chain $\alpha$ which fills $\gamma$ has $\omega(\alpha) \sim r^{3}$. This shows that $\mathrm{FV}_{H_{3}}^{2}(r) \succeq r^{3}$.

Pittet [18] generalized this technique to other nilpotent groups by using a 2dimensional subgroup in a Carnot group rather than the $y z$-plane in the Heisenberg group. He shows that if $G$ has a 2-dimensional subgroup $A$ and if there is a closed invariant 2-form $\omega$ which restricts to the area form on $A$, then one can construct cycles with large filling areas by replacing the edges of large rectangles in $A$ by geodesics. Burillo [6] generalized this argument to higher dimensions and explicitly constructed families of cycles in the Heisenberg groups to give lower bounds for their higher-order filling functions.

In the case that $G$ is a Carnot group, one can often use scaling automorphisms to help construct difficult-to-fill cycles. For example, say that $\omega$ is a closed invariant $k$-form in $G$ with the property that $s_{t}^{*}(\omega)=t^{d} \omega$. In this case, if $\alpha$ is a $k$-chain with horizontal boundary and if $\omega(\alpha) \neq 0$, then we have mass $\partial s_{t}(\alpha) \sim t^{k-1}$, but

$$
\omega\left(\left(s_{t}\right)_{\sharp}(\alpha)\right)=\left[s_{t}^{*}(\omega)\right](\alpha) \sim t^{d} .
$$

We will prove lower bounds for other nilpotent groups using this construction. We prove the following:

Theorem 9. Let $G$ be a Carnot group and let $(\tau, f: \tau \rightarrow G)$ be a triangulation of $G$. Let $\phi: \tau \rightarrow G$ be a $k$-horizontal map which is a bounded distance from $f$. Let $M$ be $a(k+1)$-dimensional subgroup of $G$. If $\omega$ is a closed invariant $(k+1)$-form on $G$ which restricts to the volume form on $M$ and which satisfies $s_{t}^{*}(\omega)=t^{d} \omega$ for all $t>0$, then

$$
\mathrm{FV}_{G}^{k+1}(V) \succ V^{d / k}
$$

Proof. We want to find a $k+1$-chain $\alpha$ such that $\partial \alpha$ is $k$-horizontal and $\omega(\alpha) \neq 0$. We start with a large ball in $M$. Let $\varepsilon>0$ be a small number to be chosen later. Since any subgroup of a nilpotent group is nilpotent, $M$ has polynomial growth, so we can choose a ball $B$ such that vol $\partial B<\varepsilon$ vol $B$. Let $[B]$ be the fundamental class of $B$, oriented so that mass $[B]=\operatorname{vol} B=\omega([B])$.

Consider the chain

$$
\alpha=Q_{\phi(\tau)}(\partial[B])+[B],
$$

where $Q_{\phi(\tau)}$ is defined as in Section 3. We have

$$
\partial \alpha=P_{\phi(\tau)}(\partial[B])-\partial[B]+\partial[B]=P_{\phi(\tau)}(\partial[B]),
$$

which is horizontal. It only remains to check that if $\varepsilon$ is sufficiently small, then $\omega(\alpha) \neq 0$. Let $c_{Q}$ be the constant from Lemma 3.1 and let

$$
\|\omega\|:=\sup \left\{\frac{|\omega(\beta)|}{\operatorname{mass} \beta}\right\},
$$


where $\beta$ ranges over all $(k+1)$-chains. Since $\omega$ is left-invariant and thus bounded, this supremum exists. If we take $\varepsilon<c_{Q}^{-1}\|\omega\|^{-1}$, then

$$
\left|\omega\left(Q_{\phi(\tau)}(\partial[B])\right)\right| \leq\|\omega\| c_{Q} \operatorname{mass} \partial[B] \leq\|\omega\| c_{Q} \varepsilon \operatorname{mass}[B]<\operatorname{mass}[B] .
$$

Thus

$$
\omega(\alpha)=\omega([B])+\omega\left(Q_{\phi(\tau)}(\partial[B])\right)>0,
$$

as desired, and the bound on the filling volume follows by the argument above.

We can use this to show that for the groups $J^{m}\left(\mathbb{R}^{k}\right)$, the upper bounds on $\mathrm{FV}_{d}$ when $d \leq k+1$ given by Theorems 6 and 8 are sharp. By Lemma 4.13, there are a triangulation $\left(\tau, f: \tau \rightarrow J^{m}\left(\mathbb{R}^{k}\right)\right)$ and a $k$-horizontal map $\phi: \tau \rightarrow J^{m}\left(\mathbb{R}^{k}\right)$ that satisfy the conditions of Theorem 9. Thus, when $d \leq k+1$, we can bound $\mathrm{FV}_{d}$ from below by finding an $d$-dimensional subgroup $M_{d} \subset J^{m}\left(\mathbb{R}^{k}\right)$ and a closed invariant $d$-form $\omega_{d}$ which restricts to the volume form of $M_{d}$.

The Lie algebra of $J^{m}\left(\mathbb{R}^{k}\right)$ can be decomposed as

$$
\dot{j}_{m, k}=\left(\mathbb{R}^{k} \oplus W_{m}\right) \oplus W_{m-1} \oplus \cdots \oplus W_{0} .
$$

Let $x_{i}: \mathbb{R}^{k} \rightarrow \mathbb{R}$ be the coordinate projections, and let $e_{1}, \ldots, e_{k} \in \mathbb{R}^{k}$ be the standard basis. Since $W_{0}$ is a 1-dimensional subspace, we can let $z: W_{0} \rightarrow \mathbb{R}$ be an isometry. Let $\mathfrak{m}_{d}=\left\langle e_{1}, \ldots, e_{d}\right\rangle$ for $i \leq k$ and $\mathfrak{m}_{k+1}=\mathbb{R}^{k} \oplus W_{0}$. These subalgebras correspond to subgroups $M_{1}, \ldots, M_{k+1}$ of $J^{m}\left(\mathbb{R}^{k}\right)$.

Let

$$
\omega_{d}=d x_{1} \wedge \cdots \wedge d x_{d}
$$

for $d \leq k$, and let

$$
\omega_{k+1}=d x_{1} \wedge \cdots \wedge d x_{k} \wedge d z .
$$

Then $\omega_{i}$ is a closed, invariant $i$-form for $1 \leq i \leq k+1$, and $\omega_{i}$ restricts to the volume form on $M_{i}$. Furthermore, $s_{t}^{*}\left(\omega_{i}\right)=t^{i} \omega_{i}$ when $i \leq k$ and $s_{t}^{*}\left(\omega_{k+1}\right)=t^{k+m} \omega_{i}$. We conclude that:

Theorem 10. $J^{m}\left(\mathbb{R}^{k}\right)$ satisfies the filling inequalities

$$
\mathrm{FV}^{i}(V) \succeq V^{\frac{i}{i-1}} \text { for } i \leq k, \quad \mathrm{FV}^{k+1}(V) \succeq V^{\frac{k+m}{k}} .
$$

In the case that $m=1$ (so that $J^{m}\left(\mathbb{R}^{k}\right)$ is a higher-dimensional Heisenberg group), this reduces to a result of Burillo [6]:

Theorem 11 (Burillo). $H_{2 k+1}$ satisfies the filling inequalities

$$
\mathrm{FV}^{i}(V) \succeq V^{\frac{i}{i-1}} \text { for } i \leq k, \quad \mathrm{FV}^{k+1}(V) \succeq V^{\frac{k+2}{k}}
$$




\section{References}

[1] D. Allcock, An isoperimetric inequality for the Heisenberg groups. Geom. Funct. Anal. 8 (1998), 219-233. Zbl 0907.22009 MR 1616147

[2] J. M. Alonso, X. Wang, and S. J. Pride, Higher-dimensional isoperimetric (or Dehn) functions of groups. J. Group Theory 2 (1999), 81-112. Zbl 0927.20021 MR 1670329

[3] G. Baumslag, C. F. Miller, III, and H. Short, Isoperimetric inequalities and the homology of groups. Invent. Math. 113 (1993), 531-560. Zbl 0829.20053 MR 1231836

[4] N. Brady, M. R. Bridson, M. Forester, and K. Shankar, Snowflake groups, PerronFrobenius eigenvalues and isoperimetric spectra. Geom. Topol. 13 (2009), 141-187. Zbl 1228.20031 MR 2469516

[5] M. R. Bridson, The geometry of the word problem. In Invitations to geometry and topology, Oxford Grad. Texts Math. 7, Oxford Univ. Press, Oxford 2002, 29-91. Zbl 0996.54507 MR 1967746

[6] J. Burillo, Lower bounds of isoperimetric functions for nilpotent groups. In Geometric and computational perspectives on infinite groups (Minneapolis, MN and New Brunswick, NJ, 1994), DIMACS Ser. Discrete Math. Theoret. Comput. Sci. 25, Amer. Math. Soc., Providence, RI, 1996, 1-8. Zbl 0849.20024 MR 1364176

[7] S. Chen, Examples of $n$-step nilpotent 1-formal 1-minimal models. C. R. Acad. Sci. Paris Sér. I Math. 321 (1995), 223-228. Zbl 0881.55011 MR 1345452

[8] D. B. A. Epstein, J. W. Cannon, D. F. Holt, S. V. F. Levy, M. S. Paterson, W. P. Thurston, Word processing in groups. Jones and Bartlett Publishers, Boston 1992. Zbl 0764.20017 MR 1161694

[9] H. Federer and W. H. Fleming, Normal and integral currents. Ann. of Math. (2) 72 (1960), 458-520. Zbl 0187.31301 MR 0123260

[10] C. Groft, Generalized Dehn functions I. Preprint, arXiv:0901.2303 [math.GR].

[11] C. Groft, Generalized Dehn functions II. Preprint, arXiv:0901.2317 [math.GR].

[12] M. Gromov, Filling Riemannian manifolds. J. Differential Geom. 18 (1983), 1-147. Zbl 0515.53037 MR 697984

[13] Geometric group theory (Sussex, 1991), vol. 2: Asymptotic invariants of infinite groups. London Math. Soc. Lecture Note Ser. 182, Cambridge University Press, Cambridge 1993. Zbl 0841.20039 MR 1253544

[14] M. Gromov, Carnot-Carathéodory spaces seen from within. In Sub-Riemannian geometry, Progr. Math. 144, Birkhäuser, Basel 1996, 79-323. Zbl 0864.53025 MR 1421823

[15] E. Leuzinger and C. Pittet, On quadratic Dehn functions. Math. Z. 248 (2004), 725-755. Zbl 1132.20021 MR 2103539

[16] V. Magnani, Contact equations, Lipschitz extensions and isoperimetric inequalities. Calc. Var. Partial Differential Equations 39 (2010), 233-271. Zbl 1195.49053 MR 2659687

[17] A. Yu. Olshanskii and M. V. Sapir, Quadratic isometric functions of the Heisenberg groups. A combinatorial proof. J. Math. Sci. 93 (1999), 921-927. Zbl 0941.20034 MR 1698761

[18] C. Pittet, Isoperimetric inequalities in nilpotent groups. J. London Math. Soc. (2) 55 (1997), 588-600. Zbl 0942.20024 MR 1452267 
[19] R. Thom, Remarques sur les problèmes comportant des inéquations différentielles globales. Bull. Soc. Math. France 87 (1959), 455-461. Zbl 0213.25302 MR 0121807

[20] B. Warhurst, Jet spaces as nonrigid Carnot groups. J. Lie Theory 15 (2005), 341-356. Zbl 1079.53062 MR 2115247

[21] S. Wenger, A short proof of Gromov's filling inequality. Proc. Amer. Math. Soc. 136 (2008), 2937-2941. Zbl 1148.53031 MR 2399061

[22] S. Wenger, Nilpotent groups without exactly polynomial Dehn function. J. Topol. 4 (2011), 141-160. Zbl 1227.20039 MR 2783380

[23] B. White, Mappings that minimize area in their homotopy classes. J. Differential Geom. 20 (1984), 433-446. Zbl 0566.57018 MR 788287

[24] R. Young, Homological and homotopical higher-order filling functions. Groups Geom. Dyn. 5 (2011), 683-690. Zbl 1263.20040 MR 2813532

[25] R. Young, Scaled relators and Dehn functions for nilpotent groups. Preprint, arXiv:math/0601297 [math.GR].

[26] R. Young, Filling inequalities for nilpotent groups. Preprint, arXiv:math/0608174 [math.GR].

Received March 25, 2011; revised February 1, 2012

R. Young, Department of Mathematics, University of Toronto, 40 St. George Street, Toronto, Ontario, M5S 2E4, Canada

E-mail: ryoung@math.toronto.edu 